\title{
Intervenciones para la prevención primaria de factores de riesgo psicosocial intralaboral: revisión sistemática de la literatura ${ }^{1}$
}

\author{
Maria Eugenia Londoño Londoño ${ }^{2}$, Héctor Felipe Cardona Ríos ${ }^{3}$, Mónica Lorena Vargas Betancur ${ }^{4}$
}

Recibido: 23/08/2016 Aceptado: 26/01/2017

DOI: $10.21772 /$ ripo.v34n2a05

\begin{abstract}
Resumen
La literatura ha documentado ampliamente la potencialidad de daño que los factores de riesgo psicosocial laborales tienen en la vida de los trabajadores. No obstante, falta claridad en las formas de intervención y el impacto de éstas. Objetivo: Caracterizar las intervenciones de prevención primaria realizadas y publicadas sobre factores de riesgo psicosocial (FRPS) intralaboral. Metodología: Este proyecto tuvo un enfoque cuantitativo tipo revisión sistemática. Se incluyeron las publicaciones de prevención primaria, incluidas en las bases de datos EBSCO, PsycNET y SciELO, en inglés, español o portugués, entre enero de 2002 y diciembre de 2011, y que documentaran los resultados obtenidos mediante la intervención. Para la clasificación de las investigaciones publicadas, seleccionadas y analizadas se utilizó una lista de chequeo y se registró en una matriz diseñada para tal fin. En las diferentes publicaciones examinadas se identificó el enfoque de las intervenciones, la población objeto y los efectos informados por cada una. Resultados: A pesar de que 2925 artículos coincidieron inicialmente con los términos de búsqueda, luego de aplicar los filtros y revisión a partir de los criterios, únicamente ocho cumplieron las reglas de inclusión. Conclusiones: En el periodo investigado existen pocas intervenciones de FRPS intralaborales de prevención primaria. La mayoría de los artículos correlacionan las características del trabajo o los individuos con las consecuencias de los FRPS y las intervenciones se dan más a nivel secundario y sobre las condiciones de los trabajadores. Los artículos seleccionados presentan intervenciones a nivel primario en las que se ve limitada su efectividad por las dificultades para mantener los cambios propuestos en el tiempo.
\end{abstract}

Palabras clave: Factores de Riesgo Psicosocial, intervención, prevención primaria, revisión sistemática de literatura.

\section{Interventions for primary prevention of intralaboral psychosocial risk factors: systematic literature review}

\begin{abstract}
The literature has extensively documented the potential damage that work's psychosocial risk factors have on the lives of workers. However, there is a lack of clarity in the forms of intervention and their impact. Objective: To characterize the primary prevention interventions conducted and published on psychosocial risk factors inside the work environment (PRF). Methodology: This project had a quantitative approach of the type systematic review. Primary prevention publications were included, found in the EBSCO, PsycNET and SciELO databases, published in English, Spanish or Portuguese between January 2002 and December 2011, which documented the results obtained by the intervention. A checklist was used for classification of the published, selected and analyzed research documents, and they were recorded in a matrix designed for this purpose. In the different publications examines the intervention focus was identified, as well as the target population and the effects reported by each one. Results: Although 2925 items initially coincided with the search terms, after applying filters and review based on the criteria, only eight met inclusion rules. Conclusions: In the period under investigation, there were a few interventions inside the work environment (PRF) of primary prevention. Most items correlate the characteristics of the work or individuals with the consequences of the PRF, and the interventions used to happen at the secondary level and on the conditions of the workers. The selected papers presented interventions at the primary level where their effectiveness is limited by the difficulties in maintaining the proposed changes over time. The literature has extensively documented the potential damage of psychosocial risk factors work on the workers' lives. However, there is a lack of clarity in the forms of intervention and their impact.
\end{abstract}

Key words: Psychosocial risk factors, intervention, primary prevention, systematic review.

1 Este trabajo de investigación se presentó para optar al título de especialistas en Salud Ocupacional de los dos primeros autores con la asesoría de la tercera. en la Universidad de Antioquia, Facultad Nacional de Salud Pública Cohorte XV

2 Centro de Investigación en Comportamiento Organizacional Cincel S.A.S.mariaeugenial@cincel.com.co

3 Universidad de Antioquia hefecari@gmail.com

4 Universidad de Antioquia lorena.vargas@udea.edu.co

Cómo citar este artículo: Londoño Londoño, M. E., Cardona Ríos, H. F., \& Vargas Betancur, M. L. (2015). Intervenciones para la prevención primaria de factores de riesgo psicosocial intralaboral: revisión sistemática de la literatura. Revista Interamericana de Psicología Ocupacional, 34(2), 120-150. Doi: 10.21772/ripo.v34n2a05 


\section{Introducción}

Los crecientes cambios en el panorama económico y laboral han evidenciado la importancia del estudio y control de los factores de riesgo psicosocial en el trabajo (FRPSL) (Aguilar \& Rentería, 2009), dado que se ha evidenciado que la globalización, el fraccionamiento de las labores, la desregularización el empleo, entre otros fenómenos, han creado condiciones que pueden llegar a tener un impacto importante en el bienestar de las personas (Schnall, Dobson \& Rosskam, 2011).

Las condiciones intralaborales abarcan las realidades propias del trabajo y su organización las cuales podrían causar potencialmente un daño en la salud y el bienestar de las personas (Ministerio de la Protección Social, 2010). Estas condiciones incluyen a aquellas directamente relacionadas con las tareas que realizan las personas, así como las características del entorno laboral tales como las características del liderazgo y de las relaciones sociales. En el mundo, los cambios en la manera de organizar el trabajo han representado un incremento en las posibilidades de que este llegue a tener efectos muy nocivos en las personas.

En primer lugar, el modelo de producción capitalista centrado fuertemente en la producción y la industrialización que tuvo lugar durante el siglo anterior, unido al modelo taylorista implicó la aparición de la tecnología de producción en serie, los procesos de trabajo muy automatizados y el trabajo a turnos (Sauter, Murphy, Hurrell, \& Levi, 2001). A su vez, la aparición de estos cambios en la estructuración del trabajo implicó la incorporación de tareas muy repetitivas, con tiempos de descanso muy cortos, una mayor exigencia en el ritmo de trabajo y menores posibilidades de que los trabajadores decidieran la manera y el orden en el que realizarían sus labores.

Por otro lado, se encuentra la globalización profundamente relacionada con las políticas y prácticas neoliberales las cuales, a su vez, han significado un aumento en la desprotección de las personas, la flexibilización del mercado laboral, el incremento de trabajos con salarios bajos y una mayor participación de las mujeres en mundo laboral (Schnall, Dobson \& Rosskam, 2011). Así, en este contexto se vuelve muy común que las personas tengan varios trabajos, que no ganen lo suficiente para satisfacer sus necesidades básicas y las de su familia, no cuenten con las condiciones mínimas de protección social y deban dedicar una mayor cantidad de tiempo a sus actividades de subsistencia (Aguilar \& Rentería, 2009).

Adicionalmente, según lo reportado por el DANE en Colombia los índices de población en edad laboral que se encuentra buscando trabajo de manera activa es cerca del 11\% (Departamento Administrativo Nacional de Estadística - DANE, 2016). Estas altas tasas de desempleo, que se viven no solamente en este país sino en otros alrededor del mundo, hacen que las personas accedan a trabajar en condiciones que atentan contra su bienestar y salud e incluso sentirse agradecidas por tener medio de subsistencia (Aguilar \& Rentería, 2009). Es así que escenarios como monotonía de las tareas, falta de definición o ambigüedad del rol, alta carga cuantitativa y cualitativa, escasas posibilidades de contar con autonomía e iniciativa en el trabajo, larga duración de la jornada laboral, trabajo nocturno, rotación y doblaje de turnos, pocos programas de capacitación y formación de los trabajadores, entre otros potencialmente dañinos se han convertido en el día a día de los trabajadores.

\section{Factores de Riesgo Psicosocial Laborales (FRPSL)}

En la literatura científica existen múltiples definiciones de los FRPSL. A continuación se presentan algunas de ellas con el fin de discutir los alcances del término.

Para la Organización Internacional del Trabajo (OIT) (Sauter et al., 2001) los FRPSL constituyen un fenómeno que comprende aspectos del puesto y 
del entorno de trabajo, como el clima o cultura de la organización, las funciones laborales, la calidad de las relaciones interpersonales y el ambiente social y el diseño y contenido de las tareas (por ejemplo, su variedad, significado, alcance, carácter repetitivo, entre otros.). También, según estos autores, el término se extiende al entorno existente fuera de la organización (por ejemplo, exigencias domésticas) y a los aspectos del individuo (por ejemplo, personalidad y actitudes) que pueden influir en la aparición del estrés en el trabajo.

Por su parte, Villalobos los FRPSL como "aquellas condiciones del individuo, del medio extralaboral o del medio laboral, que bajo determinadas condiciones de intensidad y tiempo de exposición generan efectos negativos en el trabajador o trabajadores, en la organización y en los grupos, y por último producen estrés" (Villalobos, 2007). La Resolución 2646 del 2008 del Ministerio de la Protección Social de Bogotá, Colombia, los define como "condiciones psicosociales cuya identificación y evaluación muestra efectos negativos en la salud de los trabajadores o en el trabajo"(Ministerio de la Protección Social, 2008).

Para Toro y colaboradores (Toro, Londoño, Sanin, \& Valencia, 2010) un factor de riesgo psicosocial es "una condición presente en el trabajo de una persona o de una colectividad laboral que puede afectar negativamente su salud, bienestar, desempeño y también su desarrollo personal". Esta última definición precisa efectos que no están contenidos en las anteriores, incluyendo el bienestar, el desempeño y el desarrollo personal como posibles esferas de la vida humana que potencialmente pueden ser afectadas por los factores de riesgo psicosocial.

Es común que en la práctica se confundan los términos de FRPSL y Estrés, se trata de fenómenos relacionados pero diferentes. Las condiciones del trabajo con la potencialidad de afectar la salud y el bienestar de las personas constituyen los FRPSL que también podrían ser llamados estresores o causantes de estrés. Por su parte el estrés constituye una reacción adaptativa que por condiciones de intensidad y frecuencia llega a ser negativa produciendo malestar y desordenes psicosomáticos (Caicoya, 2004).

Luego de revisar estas definiciones, se puede concluir que los FRPSL constituyen situaciones o fuentes de tipo psicológico, social y de organización del trabajo que pueden llegar a tener efectos nocivos para la salud y el bienestar de las personas. Esta definición incluye entonces aspectos intralaborales, extralaborales o externas a la organización y las condiciones individuales o características propias del trabajador (Ministerio de la Protección Social, 2010).

Sobre la naturaleza y fuentes de los factores de riesgo psicosocial laboral existen diferentes clasificaciones que proceden de observaciones directas de las condiciones psicosociales de trabajo, así como de modelos teóricos que tratan de explicar la aparición de la tensión en el trabajo y los nocivos efectos sobre la salud (OMS, 2010). Entre los más destacados están el Modelo Demanda Control (Sauter et al., 2001; Toro, 2002), al que posteriormente se le adicionó el concepto de Apoyo Social (Carver, Scheier, \& Weintraub, 1989); el Modelo Esfuerzo Recompensa (Leka, Griffiths, \& Cox, 2004), el Modelo Vitamínico de Peter Warr (Cuadra-Peralta; Veloso-Besio; Puddu-Gallardo; Salgado-García, \& Peralta-Montecinos, 2012), el Modelo Demandas-Recursos Laborales (Llorens, Bakker, Schaufeli, \& Salanova, 2007)job demands and resources evoke two relatively independent processes: health impairment and employee motivation. The robustness of the JD $\backslash \mathrm{u} 2013 \mathrm{R}$ model was tested in two different occupational samples, the first of 654 Spanish employees and the second of 477 Dutch employees. Structural equation modeling analyses provided partial evidence for the two processes. Multigroup analyses showed that the structural paths of the model were invariant across countries, although the strength of the relationships differed. We conclude that the basic structure of the JD $\backslash \mathrm{u} 2013 \mathrm{R}$ model is maintained, even when applied in different national 
and occupational contexts, when using different ways of gathering data (computerized versus paper and pencil, y el Modelo Recursos, Experiencias y Demandas (Salanova, 2009), entre otros,

Para el presente trabajo se adoptó la clasificación presentada por el Ministerio de la Protección Social en la Batería de Instrumentos para la Evaluación de los FRPSL (Ministerio de la Protección Social, 2010) la cual contempla los factores intralaborales, los extralaborales y los individuales o de condiciones personales. No obstante, es importante tener en cuenta se centró en la identificación de intervenciones específicamente sobre los FRPS intralaborales.
El Ministerio de la Protección Social (2010) agrupa las condiciones laborales cuatro grandes dominios, a saber: 1) Demandas del Trabajo, 2) Control sobre el trabajo 3) Liderazgo y relaciones sociales en el trabajo y 4) Recompensa. Cada uno de estos dominios, a su vez está dividido en una serie de dimensiones referidas a aspectos concretos del trabajo que por su configuración podrían representar un riesgo. Es importante resaltar que las intervenciones orientadas a estas dos grandes categorías apuntan a la disminución de la exposición de para la salud y bienestar de las personas. En la Tabla 1 se especifican las dimensiones que componen cada uno de los dominios mencionados.

Tabla 1. Dominios y Dimensiones de los Factores de Riesgo Psicosocial Intralaboral ${ }^{1}$

\begin{tabular}{|c|c|}
\hline DOMINIO & DIMENSIONES \\
\hline \multirow{8}{*}{$\begin{array}{l}\text { Demandas del trabajo } \\
\text { Se refieren a las exigencias que el trabajo impone al } \\
\text { individuo. }\end{array}$} & Demandas cuantitativas \\
\hline & Demandas de carga mental \\
\hline & Demandas emocionales \\
\hline & Exigencias de responsabilidad del cargo \\
\hline & Demandas ambientales y de esfuerzo físico \\
\hline & Demandas de la jornada de trabajo \\
\hline & Influencia del trabajo sobre el entorno extralaboral. \\
\hline & Consistencia de rol \\
\hline \multirow{5}{*}{$\begin{array}{l}\text { Control sobre el trabajo } \\
\text { Posibilidad que el trabajo ofrece al individuo para } \\
\text { influir y tomar decisiones sobre los diversos aspectos } \\
\text { que intervienen en su realización. }\end{array}$} & Control y autonomía sobre el trabajo \\
\hline & $\begin{array}{l}\text { Oportunidades para el uso y desarrollo de habilidades y } \\
\text { conocimientos }\end{array}$ \\
\hline & Participación y manejo del cambio \\
\hline & Claridad del rol \\
\hline & Capacitación \\
\hline \multirow{4}{*}{$\begin{array}{l}\text { Liderazgo y Relaciones sociales en el trabajo } \\
\text { El concepto de relaciones sociales en el trabajo indica } \\
\text { la interacción que se establece con otras personas en } \\
\text { el contexto laboral. }\end{array}$} & Características del liderazgo \\
\hline & Relación con los colaboradores (subordinados) \\
\hline & Retroalimentación del desempeño \\
\hline & Relaciones sociales en el trabajo \\
\hline Recompensa & Recompensas derivadas de la pertenencia a la empresa \\
\hline $\begin{array}{l}\text { Este término trata de la retribución que el trabajador } \\
\text { obtiene a cambio de sus contribuciones o esfuerzos } \\
\text { laborales. }\end{array}$ & Reconocimiento y compensación \\
\hline
\end{tabular}

1 Esta Tabla constituye una adaptación de las Tablas 1 y 2 del Documento Técnico de la Batería de Instrumentos para la Evaluación de los Factores de Riesgo Psicosocial del Ministerio de la Protección Social. 


\section{Intervención de los Factores de Riesgo Psicosocial Laboral}

Una vez los FRPSL han sido identificados, las organizaciones deben emprender acciones que busquen la eliminación o reducción de la exposición de las personas a estos factores (Moncada I Lluís, Llorens Serrano, Kristensen, \& Vega Martínez, 2005). Para guiar la intervención se encuentran dos corrientes, en primer lugar la del Instituto de Trabajo, Salud y Organizaciones $y$, en segundo lugar desde la perspectiva de la higiene ocupacional. A continuación se describen brevemente ambas.

Según el Instituto de Trabajo, Salud y Organizaciones (Leka et al., 2004) la intervención de los FRPSL debe gestionarse con un enfoque basado en la gestión del riesgo. Este enfoque parte de una valoración de los posibles riesgos en el entorno laboral y la posterior gestión y control de estos.

Al hablar de intervención de los FRPSL, dependiendo los objetivos que esta persiga y los grupos en los que se enfoque, se ha dividido en primaria, secundaria y terciaria. En la perspectiva de la higiene ocupacional la prevención primaria busca eliminar el origen del riesgo y promocionar ambientes de trabajo saludables y solidarios, la intervención secundaria busca la detección precoz de las alteraciones y realiza esfuerzos para que los trabajadores controlen su experiencia de estrésy, por último, la terciaria se orienta a la minimización de las consecuencias de la exposición a las situaciones de estrés (Martínez-Losa, \& Bestratén, 2010). Así, la prevención primaria se puede considerar un enfoque más proactivo, mientras que la secundaria y la terciaria son reactivas.

También, dependiendo del origen de los peligros que se pretenden intervenir, las acciones se pueden dividir en tres grandes categorías, a saber los individuos, la interfaz individuo-organización y la organización (Caicoya, 2004).
Las intervenciones sobre las condiciones organizacionales se centran en producir cambios en la estructura de la organización, de la tarea o el ambiente físico (Caicoya, 2004). Estas intervenciones apuntan al rediseño o cambio de las situaciones o factores que se constituyen como una fuente de FRPSL lo cual correspondería a la intervención primaria planteada anteriormente (Martínez-Losa, \& Bestratén, 2010). Algunos ejemplos de este tipo de intervención pueden ser la reducción de las exigencias cuantitativas, la redistribución de tareas, el enriquecimiento del cargo, la rotación de tareas, entre otras.

Por su parte, las intervenciones en el interfaz individuo-organización buscan mejorar las relaciones entre colegas y con los supervisores o lograr el mejor ajuste persona-medio. Esimportante resaltar que las intervenciones orientadas a estas dos grandes categorías apuntan a la disminución de la exposición de las personas a los factores de riesgo, pudiéndose incluir también en la categoría de intervención primaria.

Las intervenciones sobre el individuo pretenden brindar a las personas elementos para hacer frente a las demandas que su trabajo le impone (intervención secundaria). Esta orientación no busca cambiar las condiciones del trabajo que pueden ocasionar efectos negativos, sino cambiar las condiciones de los trabajadores para que se atenúen los efectos que esas situaciones pueden tener en ellos.

En términos de efectividad, en la literatura científica se hace un reiterado énfasis en que las intervenciones primarias o sobre las condiciones organizacionales han demostrado tener una mayor efectividad, tanto a nivel de los individuos, como a nivel de la organización (Leka et al., 2004). No obstante, la mayoría de intervenciones en las organizaciones son secundarias, se orientan al cambio de las condiciones de los individuos desarrollando herramientas para que afronten las situaciones estresantes pero ignorando la necesidad de cambiar las condiciones del entorno laboral. 
Esta deficiencia hace que la intervención resulte costosa y no sea realmente efectiva ya que hasta la persona más resistente a condiciones adversas, al estar expuesta a situaciones realmente estresantes de manera recurrente, se verá afectada en su salud y bienestar (Instituto Nacional de Seguridad e Higiene en el Trabajo, 2009; Moncada i Lluís et al., 2005).

De acuerdo con el Instituto Sindical de Trabajo, Ambiente y Salud (ISTAS), existen varios fenómenos que determinan que las intervenciones se realicen en este nivel (Lamontagne, Keegel, \& Centre, 2010; Moncada I Lluís et al., 2005):

a. Las formas de producción en el mundo actual las cuales determinan una argumentación del trabajo, la necesidad de trabajar por turnos, el afán desarrollo económico y la competitividad de las empresas se anteponen al bienestar de los trabajadores.

b. Las intervenciones no han seguido el desarrollo de los avances en el conocimiento científico. Además, la existencia de normatividad sobre el tema, que es una condición necesaria, tampoco ha sido suficiente para crear conciencia al respecto.

c. Las intervenciones tradicionalmente se han desarrollado desde el enfoque de la psicología clínica, la psicoterapia y la medicina del trabajo y estas ramas tienen un enfoque individual.

d. La concepción de que ciertos FRPSL son inherentes a determinados tipos de trabajo, lo cual hace que por definición estos se consideren estresantes y no se realicen esfuerzos por modificarlos. Sin embargo, es importante tener en cuenta que los trabajos no son así por naturaleza, sino por el diseño que se les ha dado y, por lo tanto, este enfoque puede cambiar. La excepción a esta regla serían los trabajos con altas exigencias emocionales como es el caso del personal asistencial en salud. No obstante, en estos casos puede intervenirse disminuyendo el tiempo de exposición. e. Los cambios organizacionales suelen ser más costosos y tener resultados económicos más inciertos que las intervenciones a nivel individual.

f. Generalmente las intervenciones dirigidas a los individuos suelen tener mejor calidad científica, resultados más consistentes y positivos que los que analizan las intervenciones sobre el entorno laboral organizativo (Instituto Nacional de Seguridad e Higiene en el Trabajo, 2009).

g. Los directivos de las empresas suelen atribuir la causa de los éxitos y fracasos organizacionales a las características personales de los empleados. Así, en el caso de que una persona se enferme por razón de su trabajo se considera que se da porque algo en él está mal (Dela Coleta, 2006).

En el caso de las intervenciones sobre los individuos también hace que sean menos efectivas el hecho que difícilmente se logra la participación activa de las personas cuando los empleadores no están dispuestos a cambiar las condiciones de trabajo que resultan nocivas (Instituto Sindical del Trabajo Ambiente y Salud, 2010).

En la literatura científica también es recurrente el énfasis en que la prevención primaria reporta una mayor eficacia que la secundaria y la terciaria. Esto no quiere decir que no se debe intervenir a las personas que ya han desarrollado trastornos, sino que el actuar antes de que se presente la alteración tiene un impacto más positivo en la salud y el bienestar de las personas (LaMontagne, Keegel, Louie, Ostry, \& Landsbergis, 2007). Sin embargo, lo más común en la práctica empresarial es esperar a que surjan los problemas de salud en los trabajadores para actuar, posiblemente debido al largo plazo que se requiere para ver los efectos de las intervenciones preventivas y lo difíciles de evidenciar más que las acciones terapéuticas. Aun conociendo que existen situaciones que han sido identificadas como nocivas para los grupos, se 
mantienen porque favorecen la consecución de intereses convenientes para minorías influyentes (Moncada I Lluís et al., 2005).

Otracondición importanteen lasintervenciones de los FRPSL consiste en evaluar los resultados que se logran con éstas. Es muy común que se realicen acciones y que no se valore el efecto que tienen en la salud y el bienestar de las personas e incluso en la productividad de las organizaciones (Instituto Sindical del Trabajo Ambiente y Salud, 2010). Esta es una razón por la cual los directivos de las empresas pueden estar poco dispuestos a invertir dinero en la intervención de estos factores de riesgo, porque desconocen el impacto económico que a largo plazo puede tener en su organización (Instituto Nacional de Seguridad e Higiene en el Trabajo, 2009).

\section{Revisión Sistemática de Literatura}

La revisión sistemática de la literatura es un tipo de estudio que en el caso de las ciencias sociales y humanas puede proveer un alto nivel de evidencia sobre intervenciones hechas en el área de la salud por resumir resultados de estudios disponibles y diseñados de manera cuidadosa. De acuerdo con Oakley y Fullerton (Bambra, 2009) una revisión sistemática es la exploración de una pregunta que se formula con claridad y donde se utilizan métodos sistemáticos y explícitos para identificar, seleccionar y evaluar críticamente la investigación relevante, y recopilar y analizar los datos de los estudios que se incluyen en la revisión.

Este tipo de investigaciones se diferencian de las revisiones clásicas de la literatura científica por su metodología estructurada, explícita, sistemática y multidisciplinar en la búsqueda y recolección de información, la valoración crítica de los estudios y la síntesis de lo hallado (Beltrán, 2005). Las revisiones sistemáticas se consideran estudios secundarios, pues su población de estudio la constituyen los propios estudios primarios.
Los expertos que elaboran las revisiones realizan una tarea muy metódica siguiendo los siguientes pasos (Bambra, 2009; Beltrán, 2005; Cochrane, 2016):

1. Formulación de una pregunta de investigación precisa que incluye una definición de los estudios participantes, la intervención que se hará y los resultados esperados.

2. Diseño del protocolo en el que se describen claramente los criterios de inclusión y de exclusión de los estudios, el método de consulta y las fuentes que se tendrán en cuenta.

3. Búsqueda rigurosa en las bases de datos electrónicas.

4. Selección de los estudios que cumplen estrictamente los criterios de inclusión. Es importante que en una revisión sistemática no haya un solo individuo involucrado, pues es necesario siempre una segunda revisión, y luego al reunirse comprobar los datos de extracción y darle rigurosidad metodológica y ausencia de prejuicios.

5. Extracción de los datos mediante la técnica elegida con una crítica metodológica y de síntesis de los datos para explorar mediante la descripción aquello que vaya respondiendo a la pregunta problema del estudio.

6. Valoración crítica de la calidad científica de los estudios.

7. Interpretación y reflexión sobre los datos hallados.

Se consideró que la metodología de revisión sistemática de la literatura era pertinente para el presente estudio ya que, como se mencionó anteriormente, hace falta mayor investigación precisa sobre las evidencias de intervenciones que han sido implementadas para el control de los factores de riesgo psicosocial intralaboral. Esta metodología permite agrupar los avances parciales 
que se han identificado en el tema en una fuente común con una visión general clara, completa y rigurosa, que además se mantenga actualizada, de forma que se pueda aumentar la efectividad en la toma de decisiones respecto a intervenciones sobre FRPSL en el futuro.

Teniendo en cuenta este contexto, la pretensión de este trabajo es realizar una revisión sistemática de las intervenciones publicadas en español, portugués e inglés de los FRPS de tipo intralaboral, analizando cuáles son sus enfoques, caracterizar qué clase de organizaciones las han utilizado y qué evidencias existen en relación con su eficacia en la intervención de los mismos. Se considera que solo una revisión sistemática permitiría describir datos concretos de esas metodologías publicadas.

\section{Metodología}

\section{Diseño}

Se realizó una Revisión Sistemática de tipo agregativo, cuantitativo y descriptivo, cuya unidad de análisis estuvo conformada por publicaciones en revistas indexadas que presenten resultados de investigación primaria.

\section{Procedimiento}

La búsqueda en las tres Bases de Datos Electrónicas se llevó a cabo entre los meses de abril y mayo de 2012 de manera conjunta por los investigadores.

En la Tabla 2 se encuentra la sistematización de las variables tenidas en cuenta en la revisión de los artículos finalmente seleccionados. Como unidad de análisis se tomaron las publicaciones sobre experiencias de intervención primaria de los FRPS Intralaborales. Los artículos de revista publicados en las bases de datos seleccionadas (EBSCO, PsycNET y SciELO).
Criterios de Inclusión. A continuación se presentan los criterios que fueron tenidos en cuenta para la selección de los artículos analizados. Las investigaciones debían cumplir con todos los criterios para ser incluidas.

1. Artículos científicos que describan intervenciones a nivel primario de los factores de riesgo psicosocial intralaboral. Por prevención primaria se entiende que deben estar orientadas a la reducción del riesgo en su fuente.

2. La intervención debe estar publicada en una revista científica incluida en bases de datos elegidas.

3. Idioma de publicación: inglés, español o portugués.

4. Fecha de publicación: entre enero de 2002 y diciembre de 2011.

5. La publicación debe especificar los cambios o resultados obtenidos.

\section{Criterios de exclusion.}

1. Intervenciones centradas en una sola persona, tipo caso clínico. Deben estar dirigidas a grupos de trabajadores u organizaciones.

2. Estudios que describan los efectos únicamente económicos y en productividad para la empresa y que no tenían en cuenta los efectos sobre el factor de riesgo o sobre la población de trabajadores.

3. Intervenciones con grupos de personas seleccionadas porque se les identificaron síntomas de estrés. Desde el concepto de prevención universal (Jané-llopis, 2004) al realizar el pretest se podría identificar que algunas personas reportaban niveles medios o altos de estrés, pero también otras con niveles bajos. Es decir, la muestra no fue seleccionada específicamente por sus niveles de estrés. 
Tabla 2. Sistematización de las variables examinadas en los artículos resultantes de la búsqueda sistemática

\begin{tabular}{|c|c|c|}
\hline Objetivo Especifico & Denominación & Valores posibles \\
\hline \multirow{8}{*}{$\begin{array}{l}\text { Identificar las poblaciones de } \\
\text { trabajadores en quienes se han } \\
\text { realizado las intervenciones. }\end{array}$} & $\begin{array}{l}\text { País en el que se realizó la } \\
\text { intervención. }\end{array}$ & $\begin{array}{l}\text { Estos valores serán producto de la revisión } \\
\text { sistemática. }\end{array}$ \\
\hline & Sector de la Economía & $\begin{array}{l}\text { Salud } \\
\text { Educación } \\
\text { Industrial } \\
\text { Financiero } \\
\text { Servicios } \\
\text { Minería } \\
\text { Transporte } \\
\text { Informal } \\
\text { No fue posible su identificación }\end{array}$ \\
\hline & Tamaño de la muestra & Número de personas \\
\hline & Ubicación de la muestra & $\begin{array}{l}\text { Área Rural } \\
\text { Área Urbana } \\
\text { No fue posible la identificación }\end{array}$ \\
\hline & Nivel Jerárquico & $\begin{array}{l}\text { Jefes } \\
\text { No jefes } \\
\text { Mixto } \\
\text { No fue posible la identificación. }\end{array}$ \\
\hline & Oficio & $\begin{array}{l}\text { Estos valores serán producto de la revisión } \\
\text { sistemática. }\end{array}$ \\
\hline & Género de la muestra & $\begin{array}{l}\text { Femenino } \\
\text { Masculino } \\
\text { Mixto } \\
\text { No es posible su identificación }\end{array}$ \\
\hline & Media de Edad de la muestra & Media en años \\
\hline \multirow[t]{2}{*}{$\begin{array}{l}\text { Describir los fundamentos teóricos } \\
\text { y los diseños metodológicos de las } \\
\text { intervenciones. }\end{array}$} & Enfoque teórico de la investigación & $\begin{array}{l}\text { Demanda Control } \\
\text { Demanda Control (Apoyo Social) } \\
\text { Esfuerzo-recompensa } \\
\text { Modelo Vitamínico } \\
\text { Otros }\end{array}$ \\
\hline & Metodología de la Investigación & $\begin{array}{l}\text { Estos valores serán producto de la revisión } \\
\text { sistemática de acuerdo con lo reportado por } \\
\text { los autores en su artículo. }\end{array}$ \\
\hline \multirow{2}{*}{$\begin{array}{l}\text { Identificar los FRPS intralaboral } \\
\text { objeto de las estrategias planteadas. } \\
\text { Describir los tipos de } \\
\text { intervenciones realizadas. }\end{array}$} & Foco de la Intervención & $\begin{array}{l}\text { Fuente } \\
\text { Medio } \\
\text { Individuo }\end{array}$ \\
\hline & Tipo de Intervención & $\begin{array}{l}\text { Estos valores serán producto de la revisión } \\
\text { sistemática. }\end{array}$ \\
\hline $\begin{array}{l}\text { Identificar los resultados obtenidos } \\
\text { con las intervenciones realizadas. }\end{array}$ & Variables dependientes & $\begin{array}{l}\text { Estos valores serán producto de la revisión } \\
\text { sistemática. }\end{array}$ \\
\hline $\begin{array}{l}\text { Identificar los resultados obtenidos } \\
\text { con las intervenciones realizadas. }\end{array}$ & Efectos informados & $\begin{array}{l}\text { Estos valores serán producto de la revisión } \\
\text { sistemática. }\end{array}$ \\
\hline $\begin{array}{l}\text { Identificar los FRPS intralaboral } \\
\text { objeto de las estrategias planteadas. }\end{array}$ & $\begin{array}{l}\text { Factor de Riesgo Psicosocial } \\
\text { Intralaboral intervenido }\end{array}$ & $\begin{array}{l}\text { Dimensiones plateadas en la Batería para la } \\
\text { evaluación de los factores de riesgo psicosocial } \\
\text { del Ministerio de la Protección Social. }\end{array}$ \\
\hline
\end{tabular}


Definición de las Palabras Claves. Para la elección de las Bases de datos, se tuvo presente un listado de cinco bases de datos sobresalientes en el tema de Revisiones Sistemáticas, y luego de consultar con las personas expertas en bibliotecología determinamos incluir las tres bases de datos más orientadas a psicología y salud ocupacional: EBSCOhost: Psychology and Behavioral Sciences Collection, APA PsycNET (PsycInfo) y SciELO: Scientific Electronic Library Online (Biblioteca Cientifica Electrónica en Linea).

Para la definición de estas palabras clave, los investigadores realizaron una consulta bibliográfica con el fin de identificar los términos utilizados más frecuentemente en la literatura científica sobre FRPSL e intervenciones dirigidas a atenderlos. Con base en esta revisión realizamos una consulta de los términos descriptores equivalentes en Ciencias de la Salud $(\mathrm{DeCS})^{2}$ pero muy pocos tuvieron equivalencia y los que lo hicieron no abarcaban el tema y no incluían el término psicosocial. Los términos DeCS, que estaban disponibles en el idioma inglés encontrados fueron: prevention and control, social support, burnout, work environment, ergonomics, cognitive ergonomics, risk, risk factor, work load, workflow, job, organization, company, labor, workplace, work-place, occupational. Por esta razón se tomó la decisión de incluir en la búsqueda los términos DeCS las palabras que aparecían de manera recurrente en la revisión inicial a pesar de que esto supusiera que las consultas en las bases de datos arrojaran un número grande de artículos que no cumplieran con los criterios de inclusión.

Con base en la revisión de literatura inicial, se consideró que éstas podrían dividirse en tres grupos para realizar la búsqueda: (a) ¿qué se hace?

2 La consulta de los términos Decs se realizó en http://decs.bvs.br/ incluyó las palabras equivalentes a intervención o modificación de las condiciones laborales; (b) ¿sobre qué se interviene? que agrupa las palabras referidas a los aspectos que generalmente son objeto de intervención, y, (c) ¿đónde se interviene? para limitar los artículos al entorno laboral.

Es importante aclarar que, a pesar de que en los criterios de inclusión se decidió tener en cuenta también los artículos escritos en portugués, no se definieron palabras clave en este idioma dado que la mayoría de las publicaciones presentan su abstract en dos idiomas, de tal manera que se identificarían los artículos escritos en portugués por su resumen en inglés o español.

Proceso de Búsqueda. El método de búsqueda para cada una de las bases de datos seleccionadas es diferente, sin embargo, se realizó en las tres la búsqueda con las palabras clave definidas en Inglés, puesto que, al ser revistas internacionales, cada estudio debe tener el abstract o resumen en este idioma. Además, algunas palabras compuestas debieron ser encerradas entre comillas para que los resultados fueran más precisos.

En las tres bases de datos se seleccionó la opción de "búsqueda avanzada", lo que permitió reunir los términos de acuerdo a las categorías anteriormente mencionadas ¿Qué se hace? ¿Sobre qué se interviene? ¿Dónde se interviene? y con la ayuda de los "conectores booleanos": "OR" "AND".

Proceso de búsqueda general. En la Figura 1 se pueden observar los pasos seguidos para la realización de la búsqueda en las bases de datos bibliográficas y a continuación se explica cada una de estas fases. Inicialmente el número de hallazgos de las tres bases de datos en la primera búsqueda fue de 2925 artículos que coincidían aparentemente con los criterios definidos. 
Tabla 3. Grupos de palabras clave establecidas para la bísqueda en bases de datos bibliográficas

\begin{tabular}{|c|c|c|}
\hline ¿Qué se hace? & ¿Sobre qué se interviene? & ¿Dónde se interviene? \\
\hline $\begin{array}{l}\text { Español: buenas prácticas, } \\
\text { intervención, administración, } \\
\text { Protección, Optimización, } \\
\text { Programa de prevención, } \\
\text { Intervención preventiva, } \\
\text { Intervención primaria, } \\
\text { Intervención preventiva } \\
\text { primaria, Administración } \\
\text { del riesgo, Tratamiento del } \\
\text { riesgo, Reorganización, } \\
\text { Prevención y Control. }\end{array}$ & $\begin{array}{l}\text { Español: Control, Demanda, A nivel del } \\
\text { empleador, Desbalance esfuerzo-recompensa } \\
\text { (retribución), Salud Mental, A nivel de la } \\
\text { organización- A nivel organizacional, Factores } \\
\text { positivos, Psicosocial, Entorno Psicosocial, } \\
\text { Factor Psicosocial, Salud Psicosocial, Riesgo } \\
\text { Psicosocial, Factor de riesgo psicosocial, } \\
\text { Apoyo social, Tensión, estrés laboral, Estrés, } \\
\text { Estresores-Factores de estrés, Reestructuración } \\
\text { de la tarea, Estructura de la tarea, Dirigida } \\
\text { al trabajador, Condiciones de trabajo, } \\
\text { Ambiente de trabajo, Condiciones de Trabajo, } \\
\text { Ergonomía, Ergonomía Cognitiva, Riesgo, } \\
\text { Factores de Riesgo, Carga de Trabajo, Flujo de } \\
\text { Trabajo. }\end{array}$ & $\begin{array}{l}\text { Español: Trabajo, } \\
\text { Empleo, Organización, } \\
\text { Compañía, Labor, Sitio } \\
\text { de trabajo-Lugar de } \\
\text { Trabajo, Ocupacional. }\end{array}$ \\
\hline $\begin{array}{l}\text { Inglés: good practice, } \\
\text { intervention, Management, } \\
\text { Protection, optimization, } \\
\text { prevention programs, } \\
\text { preventive interventions, } \\
\text { primary intervention, } \\
\text { primary preventive } \\
\text { interventions, risk } \\
\text { management, risk treatment, } \\
\text { reorganization, prevention } \\
\text { and control. }\end{array}$ & $\begin{array}{l}\text { Inglés: control, demand, employer level, } \\
\text { effort-reward imbalance, Mental Health, } \\
\text { organizational level, positive factors, } \\
\text { Psychosocial, psychosocial environment, } \\
\text { psychosocial factors , psychosocial health, } \\
\text { psychosocial risk, psychosocial risk factors, } \\
\text { social support, strain, burn out, stress, } \\
\text { stressors, task restructuring, task structure, } \\
\text { work-directed, working conditions, } \\
\text { work environment, working conditions, } \\
\text { ergonomics, cognitive ergonomics, risk, risk } \\
\text { factors, workload, workflow. }\end{array}$ & $\begin{array}{l}\text { Inglés: work, job, } \\
\text { organization, company, } \\
\text { labor, workplace, work- } \\
\text { place, occupational. }\end{array}$ \\
\hline
\end{tabular}

Nota: A pesar de que en los criterios de inclusión contenían los artículos escritos en portugués, no se definieron palabras clave en este idioma dado que la mayoría de las publicaciones presentan su abstract en dos idiomas, de tal manera que se identificarían los artículos escritos en portugués por su resumen en inglés o español. 


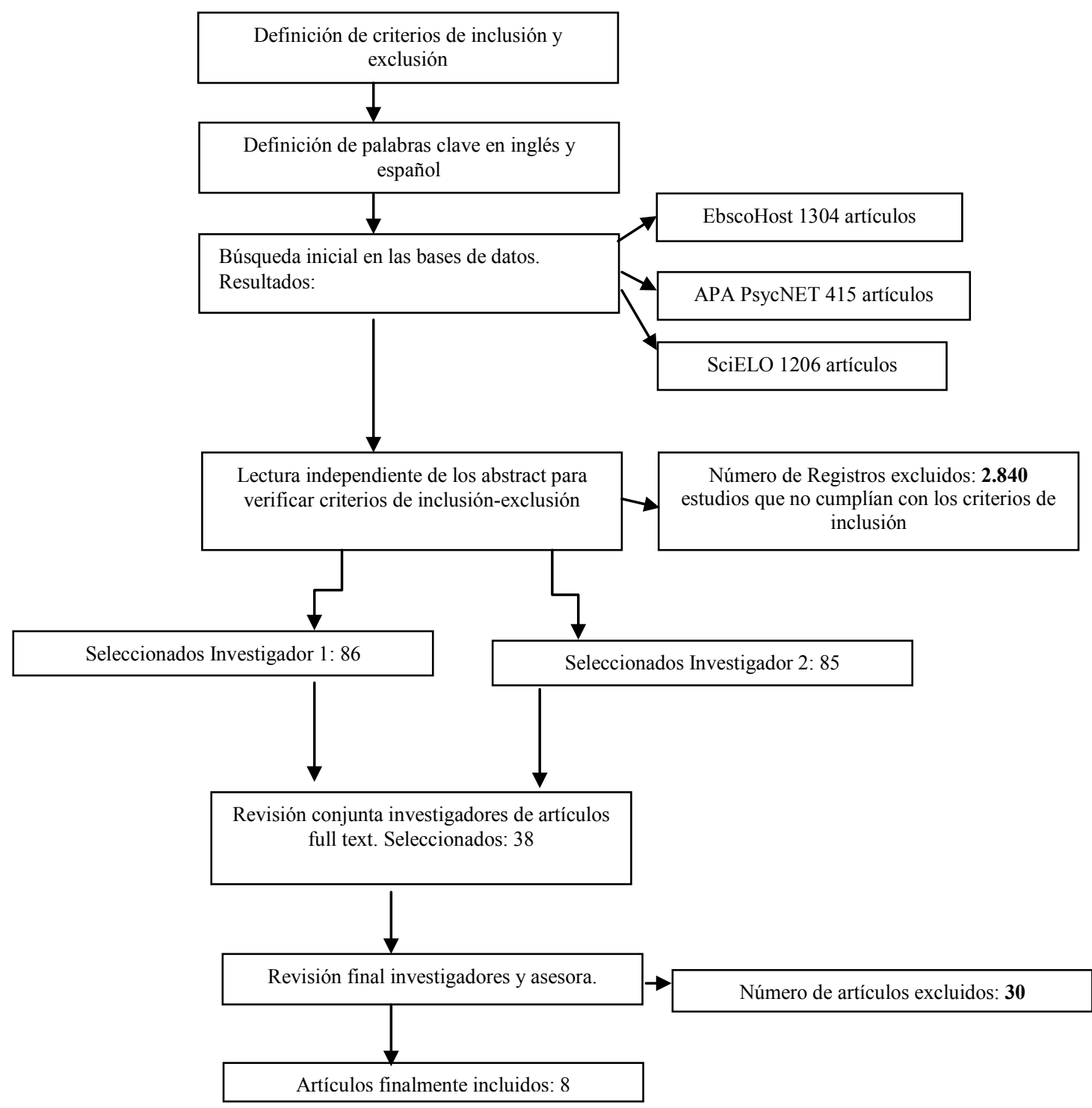

Figura 1. Proceso general de búsqueda.

Después de la búsqueda y lectura de los abstract entre ese número de artículos por cada investigador de manera independiente, se seleccionaron 86 artículos por parte del investigador 1 y 85 artículos por el segundo.

Posteriormente estos dos investigadores se reunieron para revisar de manera conjunta los artículos preseleccionados en la búsqueda independiente. La mayoría de ellos fueron seleccionados en común. No obstante, luego de realizar una lectura conjunta de los abstract y, en caso de ser necesario una revisión del texto completo, se seleccionaron finalmente 38 artículos (Full text).

Se realizó una última verificación de manera conjunta con la tercera investigadora con el fin de clarificar con precisión el nivel en el que los artículos cumplían con los criterios de inclusión y exclusión. En este paso eliminaron 30 artículos. Las razones de exclusión fueron las siguientes: 
- No estaban referidos a intervenciones sobre Factores de Riesgo Psicosocial Intralaboral o correlacionaban fenómenos (mediciones, encuestas, descripciones, relaciones teóricas entre factores): 11 estudios excluidos.

- No se referían a intervenciones exclusivamente sobre Factores de Riesgo Psicosociales intralaborales sino que se trataba de intervenciones sobre factores de riesgos psicosocial personales, cinco estudios excluidos

- Se orientaban a la intervención de los efectos de los factores de riesgo psicosocial 14 estudios excluidos.

Finalmente se aprobaron ocho artículos científicos que apuntan a los objetivos y cumplen con los criterios de inclusión propuestos en el diseño metodológico. Vale la pena mencionar que, a pesar de que en los criterios de inclusión se contemplaron artículos en portugués, ninguno de los analizados finalmente está publicado en este idioma.

Aspectos éticos de la investigación. De acuerdo con los criterios establecidos en la Resolución 8430 de 1994 del Ministerio de Salud de Colombia, en su numeral 11 los estudios que emplean métodos de investigación documental retrospectivos como es el caso del presente proyecto, y aquellos en los que no se realiza ninguna intervención o modificación intencionada de las variables biológicas, fisiológicas, psicológicas o sociales de los individuos que participan en el estudio, son considerados como estudios sin riesgo para los seres humanos.

\section{Resultados}

\section{Evaluación de la calidad de los artículos finalmente seleccionados}

Con el fin de evaluar de una manera estructurada y objetiva la calidad investigativa de los artículos finalmente seleccionados se realizó un análisis basado en la metodología planteada por Effective Public Health Practice Project (Thomas, 2003). Es una herramienta para que los investigadores consideren varios aspectos metodológicos de los artículos y puedan identificar posibles sesgos que alteren los resultados. Los resultados de esta evaluación pueden consultarse en la Tabla 4.

En primer lugar, se analizó el sesgo de selección (Criterio A), referido a la representatividad que tiene la muestra seleccionada, en este caso, estudios, de la población total (institución, oficio, sector) y el grado de acuerdo que mostraron las personas elegidas para participar en el estudio. Tres de los estudios fueron calificados por los evaluadores con un control débil del sesgo de selección y los cinco restantes como moderados. Esto se debe a que en varios de ellos no se especificó el tamaño de la población total y, por lo tanto, no fue posible identificar la representatividad de la muestra. De la misma manera en los que fueron calificados como débiles se mencionó cuál fue la muestra con la que se trabajó, pero no se especificó cuántos individuos fueron invitados inicialmente a participar y qué porcentaje de ellos estuvieron de acuerdo en hacerlo.

En la evaluación de diseño del estudio (Criterio B) se revisó la probabilidad de sesgo debido al tipo de diseño experimental utilizado. En esta metodología se consideran como fuertes los estudios en los que se tiene un grupo control y el proceso de asignación es tal que los investigadores no podrían predecir en cuál de los grupos estará finalmente la persona. En este aspecto la totalidad de los estudios se ubicaron en el rango moderado, siendo favorecida esta calificación por el hecho de que todas las investigaciones contaron con mediciones antes y después de la intervención que se realizó.

No obstante, como debilidad en este aspecto se encontró que tres de ellos no contaron con un grupo de control lo cual hace que se aumente la posibilidad de sesgo al momento de interpretar los resultados. En los cinco estudios que sí incluyeron 
un grupo de control, la calificación se afectó por el hecho de que no se especificó si la asignación a los grupos fue aleatoria ni los métodos por medio de los cuales se determinó en qué grupos estarían los individuos.

A continuación, se calificaron los artículos en relación al manejo que hicieron de los factores de confusión o variables que pueden estar asociadas a la exposición y tener una relación causal con el resultado de interés (Criterio $\mathrm{C}$ ). En este caso se consideran como fuertes los artículos en los que los autores informaron que tuvieron en cuenta la presencia de factores de confusión y, en caso de encontrarse, si estos fueron controlados. Si la asignación a los grupos de intervención y de control es al azar, se espera que los autores informen que se aseguraron de que los grupos fueron equilibrados respecto de los factores de confusión antes de comenzar la intervención. En este caso cinco de los estudios fueron calificados como fuertes y tres con un débil control.

$\mathrm{Al}$ evaluar el factor de Cegamiento (Criterio D) en los estudios todos fueron calificados como débiles. Esto se debe a que en cada una de las intervenciones que utilizaron grupo control los investigadores conocían en cuál de los dos grupos se encontraba cada individuo y en ninguno de los estudios seleccionados se especificó si los participantes conocían la pregunta de investigación.

En relación con los instrumentos utilizados para la recolección de la información (Criterio E), siete de los estudios fueron calificados como fuertes debido a que reportaban niveles altos de calidad de los instrumentos en relación a la su validez y confiabilidad. El estudio restante no cumplió con estos requisitos y fue calificado como débil.

$\mathrm{Al}$ analizar la calidad de los estudios en la sección referida a los retiros y abandonos (Criterio F) se encontró que tres de ellos informaron de manera clara la cantidad de retiros por cada uno de los grupos de las muestras seleccionadas, por lo cual se calificaron como fuertes. Cuatro fueron calificados como moderados y solo uno como débil.

Finalmente, en la calificación global de los artículos cinco de ellos fueron calificados como débiles y los tres restantes como moderados. 
Tabla 4. Evaluación de la calidad de los artículos seleccionados finalmente

\begin{tabular}{|c|c|c|c|c|c|c|c|}
\hline Nombre del artículo & A & $\mathrm{B}$ & $\mathrm{C}$ & $\mathrm{D}$ & $\mathrm{E}$ & $\mathrm{F}$ & $\begin{array}{c}\text { Puntaje } \\
\text { global }\end{array}$ \\
\hline $\begin{array}{l}\text { Assessing the impact of healthy work organization } \\
\text { intervention (DeJoy, Wilson, Vandenberg, } \\
\text { McGrath-Higgins, \& Griffin-Blake, 2010) }\end{array}$ & Moderado & Moderado & Fuerte & Débil & Fuerte & Moderado & Moderado \\
\hline $\begin{array}{l}\text { Time Management Training and Perceived } \\
\text { Control of Time at Work (Häfner \& Stock, 2010) }\end{array}$ & Débil & Moderado & Débil & Débil & Fuerte & Moderado & Débil \\
\hline $\begin{array}{l}\text { Evaluation of an intervention programme based } \\
\text { on empowerment for eldercare nursing staff } \\
\text { (Petterson, Donnersväärd, Lagerströöm, \& } \\
\text { Toomingas, 2006) }\end{array}$ & Moderado & Moderado & Fuerte & Débil & Fuerte & Moderado & Moderado \\
\hline $\begin{array}{l}\text { Effects of resident-oriented care on job } \\
\text { characteristics of nursing caregivers (J. M. B. } \\
\text { Berkhout, P. G. Boumans, J. N. Nijhuis, P. J. Van } \\
\text { Breukelen, \& Huijer Abu-saad, 2003) }\end{array}$ & Débil & Moderado & Fuerte & Débil & Fuerte & Moderado & Débil \\
\hline $\begin{array}{l}\text { Evaluation of an educational "toolbox" for } \\
\text { improving nursing staff competence and } \\
\text { psychosocial work environment in elderly } \\
\text { care: Results of a prospective, non-randomized } \\
\text { controlled intervention (Arnetz \& Hasson, 2007) }\end{array}$ & Moderado & Moderado & Débil & Débil & Fuerte & Débil & Débil \\
\hline $\begin{array}{l}\text { Efecto de una intervención psicoeducativa para } \\
\text { disminuir el Síndrome Burnout en personal de } \\
\text { confianza de la Comisión Federal de Electricidad } \\
\text { (Pivaral, Cruz, Pérez, López, \& Figueroa, 2009) } \\
\text { bringing about stressing situations. All those events } \\
\text { perceived as threatening bringing about tension are } \\
\text { called stressors. A stressing situation comes about } \\
\text { when there existsadiscrepancybetween environment } \\
\text { demands and adequate resources to cope with them. } \\
\text { All these factors lead to physiological, cognitive } \\
\text { and motor responses which enhance perception } \\
\text { and create the need of better solutions to allow } \\
\text { appropriate adaptation behaviors. When stress is } \\
\text { present at the workplace in a recurrent fashion, it is } \\
\text { known as Labor Stress (LS }\end{array}$ & Moderado & Moderado & Fuerte & Débil & Fuerte & Fuerte & Moderado \\
\hline $\begin{array}{l}\text { Stretching and joint mobilization exercises reduce } \\
\text { call-center operators' musculoskeletal discomfort } \\
\text { and fatigue (Lacaze, Sacco, Rocha, Pereira, \& } \\
\text { Casarotto, 2010) }\end{array}$ & Débil & Moderado & Fuerte & Débil & Débil & Fuerte & Débil \\
\hline $\begin{array}{l}\text { Evaluation of Training Designed to Prevent and } \\
\text { Manage Patient Violence (Calabro, Mackey, \& } \\
\text { Williams, 2002) }\end{array}$ & Moderado & Moderado & Débil & Débil & Fuerte & Fuerte & Débil \\
\hline
\end{tabular}

Nota. Columna A: Calificación del estudio en relación con el Sesgo de Selección, Columna B: Calificación del estudio en relación con el Diseño del estudio, Columna C: Calificación del estudio en relación con los Factores de Confusión, Columna D: Calificación del estudio en relación con el Cegamiento, Columna E: Calificación del estudio en relación con el Proceso para la recolección de a información, Columna F: Calificación del estudio en relación con los Retiros y abandonos (Tomado de: Effective Public Health Practice Project (56)). 


\section{Análisis del contenido de los artículos seleccionados}

En primer lugar, es importante destacar que se encontró un número reducido de artículos que cumplieran con los criterios de inclusión que se definieron para este trabajo (ocho artículos). A pesar de que el número de artículos que inicialmente apareció en las bases de datos consultadas con las palabras clave definidas fue muy extenso (2925), en las revisiones realizadas se encontró que la mayoría de ellos eran correlacionales, se centraban en establecer relaciones entre condiciones del trabajo o de los individuos y algunos efectos sobre la salud y el bienestar (Hernández, Fernández, \& Baptista, 2010), en la intervención secundaria, es decir en el tratamiento de los efectos de los FRPSL o en la intervención de las condiciones personales.

\section{Poblaciones de trabajadores en quienes se han realizado las intervenciones}

En la Tabla 5 se pueden observar las variables de las muestras utilizadas en los estudios que fueron tenidas en cuenta por cumplir los criterios de inclusión. De los ocho estudios identificados, cuatro de ellos fueron realizados en Europa (Suecia, Holanda, Alemania), dos en Estados Unidos y los dos restantes en Latinoamérica. Todos los estudios fueron realizados con población urbana, ninguno incluyó trabajadores rurales.
Al revisar el oficio de las personas que conformaban las muestras en los estudios se encuentra que la mitad de ellos fueron realizados con personal asistencial en salud. Entre los demás oficios incluidos se encuentra uno con operadores de centros de llamadas (call centers) y, finalmente, uno con vendedores. El estudio restante, se realizó en una empresa comercial, no obstante, no especificó qué clase de oficios realizaban las personas que participaron en la intervención.

En lo referente al sector de la economía, se encuentran resultados muy relacionados con lo mencionado en los oficios, cuatro de los estudios fueron realizados en el sector salud, dos en empresas dedicadas a la comercialización de productos, uno en una organización de tipo industrial y otro en una empresa dedicada a la atención telefónica de usuarios.

$\mathrm{Al}$ analizar los resultados por nivel jerárquico, se encuentra que cinco de los estudios no especifican claramente si la muestra incluía exclusivamente a personas sin mando o también a jefes. La muestra de un estudio estuvo constituida por un grupo de líderes de una empresa industrial, uno con personal sin responsabilidades de dirección y uno con una muestra mixta.

En relación con el género de las muestras intervenidas, siete estudios fueron realizados con hombres y mujeres, mientras que el restante tuvo una muestra exclusivamente femenina. 
Tabla 5. Caracteristicas de las muestras en las que se realizaron las intervenciones

\begin{tabular}{|c|c|c|c|c|c|c|c|c|c|c|c|c|}
\hline$\stackrel{\dot{\theta}}{\dot{\infty}}$ & 总足 & Nombre Artículo & Autores & Revista & Año & País & 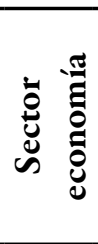 & 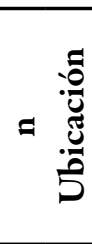 & 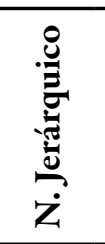 & $\underbrace{\circ}_{0}$ & 递 & 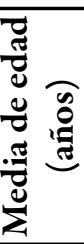 \\
\hline 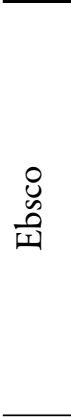 & 1 & $\begin{array}{l}\text { Assessing the } \\
\text { impact of healthy } \\
\text { work organization } \\
\text { intervention }\end{array}$ & $\begin{array}{l}\text { David M. } \\
\text { DeJoy, Mark G. } \\
\text { Wilson, Robert } \\
\text { J. Vandenberg, } \\
\text { Allison L. } \\
\text { McGrath- } \\
\text { Higgins and } \\
\text { C. Shannon } \\
\text { Griffin-Blake } \\
\end{array}$ & $\begin{array}{c}\text { Journal of } \\
\text { Occupatio } \\
\text { nal and } \\
\text { Organiza } \\
\text { tionalPsycho } \\
\text { logy }\end{array}$ & $\stackrel{\circ}{\circ}$ & 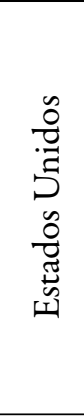 & 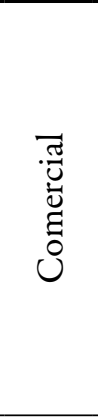 & 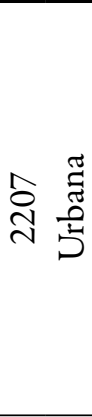 & 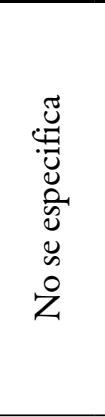 & 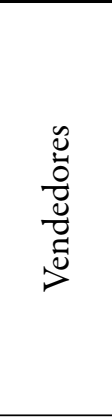 & 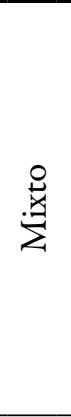 & $\stackrel{m}{m}$ \\
\hline $\begin{array}{c}\stackrel{\mathscr{U}}{0} \\
\stackrel{0}{0}\end{array}$ & 2 & $\begin{array}{l}\text { Time Management } \\
\text { Training and } \\
\text { Perceived Control } \\
\text { of Time at Work }\end{array}$ & $\begin{array}{l}\text { A. Hafner \& A. } \\
\text { Stock }\end{array}$ & $\begin{array}{l}\text { The Journal of } \\
\text { Psychology }\end{array}$ & $\stackrel{\circ}{\stackrel{i}{N}}$ & 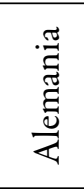 & 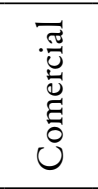 & 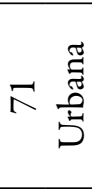 & 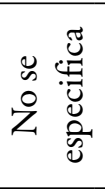 & 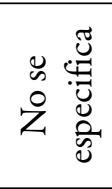 & 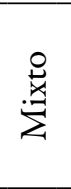 & $n$ \\
\hline $\begin{array}{c}\stackrel{0}{u} \\
\stackrel{0}{0} \\
1\end{array}$ & 3 & $\begin{array}{l}\text { Evaluation of } \\
\text { an intervention } \\
\text { programme based } \\
\text { on empowerment } \\
\text { for eldercare } \\
\text { nursing staff }\end{array}$ & $\begin{array}{l}\text { I.-L. Petterson, } \\
\text { H. Donnersva, } \\
\text { M. Lagerstro, } \\
\text { M. Toomingas }\end{array}$ & Work and Stress & ஓ̊ & 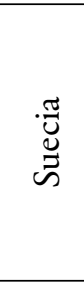 & $\frac{\overrightarrow{3}}{n}$ & 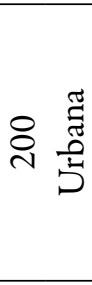 & 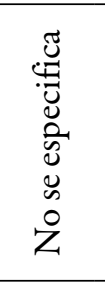 & 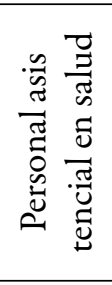 & $\stackrel{\stackrel{*}{*}}{\stackrel{*}{\Sigma}}$ & 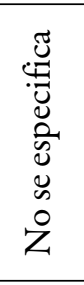 \\
\hline $\begin{array}{c}\stackrel{\mathscr{U}}{0} \\
\stackrel{0}{0}\end{array}$ & 4 & $\begin{array}{l}\text { Effects of resident- } \\
\text { oriented care on } \\
\text { job characteristics } \\
\text { of nursing } \\
\text { caregivers }\end{array}$ & $\begin{array}{l}\text { Afke J. M. } \\
\text { B. Berkhout, } \\
\text { Nicolle P.G. } \\
\text { Boumans, Frans } \\
\text { J. N. Nijhuis, } \\
\text { Gerard P. J. Van } \\
\text { Breukelen and } \\
\text { Huda Huijer } \\
\text { Abu-Saad }\end{array}$ & Work and Stress & ֻి & $\begin{array}{l}\frac{\pi}{3} \\
\frac{0}{0} \\
\text { I }\end{array}$ & $\frac{\overrightarrow{0}}{\tilde{\pi}}$ & 유류 & 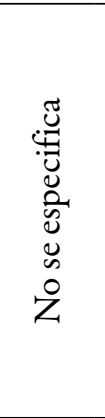 & 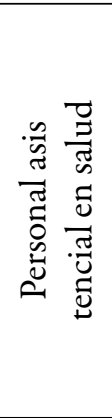 & 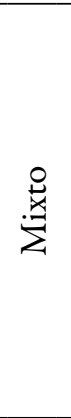 & 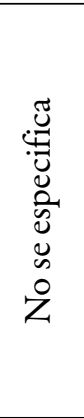 \\
\hline 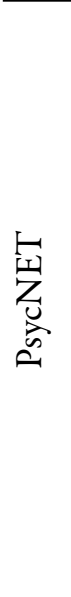 & 5 & $\begin{array}{l}\text { Evaluation of } \\
\text { an educational } \\
\text { "toolbox" for } \\
\text { improving nursing } \\
\text { staff competence } \\
\text { and psychosocial } \\
\text { work environment } \\
\text { in elderly care: } \\
\text { Results of a } \\
\text { prospective, non- } \\
\text { randomized } \\
\text { controlled } \\
\text { intervention }\end{array}$ & $\begin{array}{l}\text { J.E. Arnetza \& } \\
\text { H. Hassona }\end{array}$ & $\begin{array}{c}\text { International } \\
\text { Journal of } \\
\text { Nursing Studies }\end{array}$ & हิ & 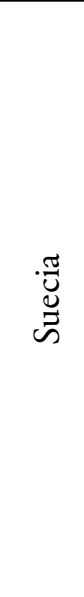 & $\frac{\vec{D}}{\vec{J}}$ & 尽莡 & $\stackrel{\stackrel{\circ}{*}}{\stackrel{*}{\Sigma}}$ & 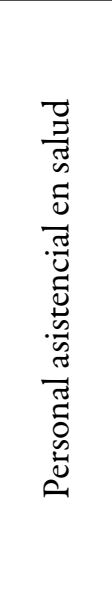 & $\stackrel{\stackrel{\circ}{*}}{\stackrel{*}{\Sigma}}$ & 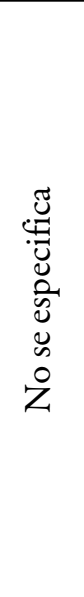 \\
\hline
\end{tabular}




\begin{tabular}{|c|c|c|c|c|c|c|c|c|c|c|c|}
\hline$\dot{\varphi}:$ & $\stackrel{\circ}{: 0}$ & Nombre Artículo & Autores & Revista & Año & País & 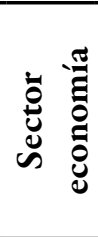 & 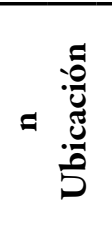 & 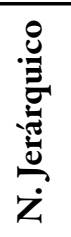 & : & 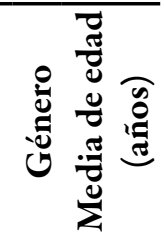 \\
\hline 号 & 6 & $\begin{array}{l}\text { Efecto de una } \\
\text { intervención } \\
\text { psicoeducativa } \\
\text { para disminuir el } \\
\text { Síndrome Burnout } \\
\text { en personal de } \\
\text { confianza de la } \\
\text { Comisión Federal } \\
\text { de Electricidad }\end{array}$ & $\begin{array}{l}\text { C. Cabrera, } \\
\text { L.K. Ruiz, } \\
\text { G.J. González, } \\
\text { M.G. Vega \& I. } \\
\text { Valadez }\end{array}$ & Salud Mental & 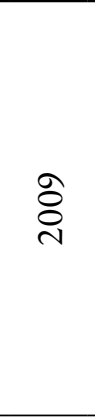 & 苟 & 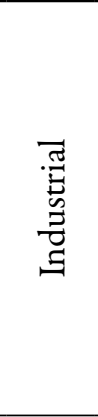 & 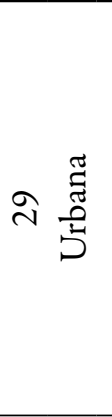 & 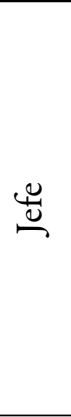 & 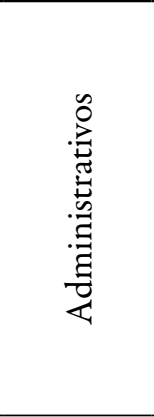 & 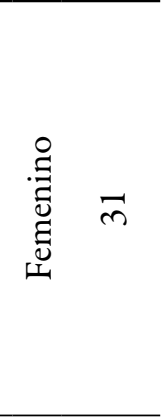 \\
\hline 足 & 7 & $\begin{array}{l}\text { Stretching and } \\
\text { Joint Mobilization } \\
\text { Exercises Reduce } \\
\text { Call-Center } \\
\text { Operators } \\
\text { Musculoskeletal } \\
\text { Discomfort and } \\
\text { Fatigue }\end{array}$ & $\begin{array}{l}\text { D.H de } \\
\text { Castro, I.C. } \\
\text { N. Sacco, L.E. } \\
\text { Rocha, C.A. } \\
\text { de Bragança \& } \\
\text { R.A. Casarotto }\end{array}$ & Clinical Science & $\stackrel{\circ}{\stackrel{0}{0}}$ & 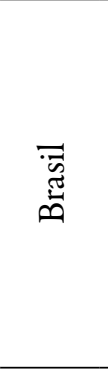 & 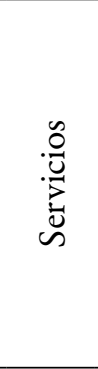 & ث艹 & 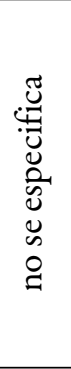 & 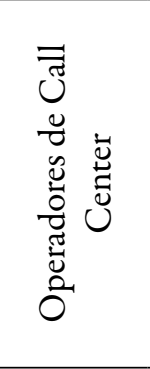 & 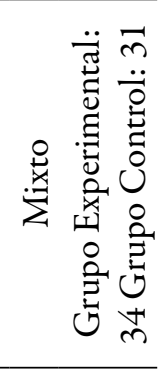 \\
\hline 莕 & 8 & $\begin{array}{l}\text { Evaluation of } \\
\text { Training Designed } \\
\text { to Prevent and } \\
\text { Manage Patient } \\
\text { Violence }\end{array}$ & $\begin{array}{l}\text { K. Calibri, T. } \\
\text { A. Mackey, } \\
\text { S.Williams }\end{array}$ & $\begin{array}{l}\text { Issues in Mental } \\
\text { Health Nursing }\end{array}$ & §్̊ & 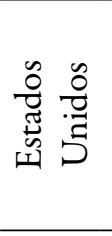 & $\frac{\vec{T}}{\stackrel{5}{\pi}}$ & 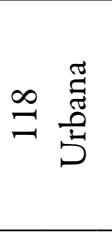 & $\frac{\stackrel{\mathscr{U}}{\tilde{U}}}{\stackrel{\Xi}{g}}$ & 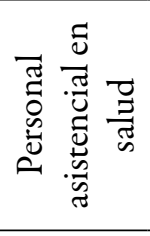 & 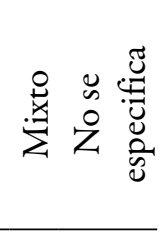 \\
\hline
\end{tabular}

En lo referente con la edad se dificultó la descripción de las muestras utilizadas ya que se describían con criterios diferentes como rangos o promedios. En el diseño del presente estudio se determinó trabajar con la media de edad de la muestra, no obstante, solamente en la mitad de los estudios se especificó ésta, encontrándose que en todas las investigaciones en la que se reportan la edad de los participantes la media se encuentra entre los 30 y los 40 años.

\section{En relación con la fundamentación teórica y los diseños metodológicos.}

En la Tabla 6 se establece el modelo desde el que se entiende que aparecen las condiciones necesarias para que se considere que existen FRPSL. Es importante anotar que en algunas de ellas no se mencionó abiertamente el enfoque utilizado y éste por lo tanto se infirió a partir del marco teórico de cada estudio.

Los artículos numerados del 2 al 8 se basaron en el Modelo de Demanda-Control de Karasek y en cuatro de ellos se consideró el apoyo social como una variable complementaria de este modelo. Únicamente un estudio se basó en la teoría de las Organizaciones Saludables.

En el estudio número 6, a pesar de que el título puede sugerir que se trata de una intervención secundaria, los investigadores decidieron incluirlo dado que la muestra fue elegida al azar entre los directivos de la empresa en la que se realizó. Además porque la preprueba se identificaron personas con síntomas de burnout y otras sin ellos, lo cual se adapta a criterio de prevención primaria universal (Jané-llopis, 2004). 
Tabla 6. Diseños Metodológicos y fundamentación teórica de los estudios hallados

\begin{tabular}{|c|c|c|}
\hline $\begin{array}{l}\text { Código } \\
\text { Artículo }\end{array}$ & Enfoque teórico & Diseño \\
\hline 1 & $\begin{array}{l}\text { Modelo de Organizaciones } \\
\text { Saludables }\end{array}$ & $\begin{array}{l}\text { Investigación longitudinal (con grupo control) (medida pretest y } \\
\text { postest con pruebas escritas) }\end{array}$ \\
\hline 2 & $\begin{array}{l}\text { Modelo Demanda Control } \\
\text { de Karasek }\end{array}$ & Diseño experimental de 2 (tratamiento-Control) ${ }^{*} 2$ (pretest-postest) \\
\hline 3 & $\begin{array}{l}\text { Modelo Demanda Control } \\
\text { de Karasek (apoyo social). }\end{array}$ & Diseño prospectivo (medida pretest y postest con pruebas escritas) \\
\hline 4 & $\begin{array}{l}\text { Modelo Demanda Control } \\
\text { de Karasek (apoyo social) }\end{array}$ & $\begin{array}{l}\text { Un diseño de pre-test post-test con grupo control (medida pretest y } \\
\text { postest con pruebas escritas y entrevistas semiestructuradas) }\end{array}$ \\
\hline 5 & $\begin{array}{l}\text { Modelo Demanda Control } \\
\text { de Karasek (apoyo social). }\end{array}$ & $\begin{array}{l}\text { Estudio prospectivo, no aleatorizado, controlado; con aplicaciones de } \\
\text { test pre-intervención y } 2 \text { post intervención }\end{array}$ \\
\hline 6 & $\begin{array}{l}\text { Modelo Demanda Control } \\
\text { de Karasek }\end{array}$ & $\begin{array}{l}\text { Estudio cuasi experimental con una medición pre y post a la } \\
\text { intervención educativa. }\end{array}$ \\
\hline 7 & $\begin{array}{l}\text { Modelo Demanda Control } \\
\text { de Karasek }\end{array}$ & $\begin{array}{l}\text { Estudio piloto longitudinal (Grupo Control y Grupo Experimental) } \\
\text { con medición una vez por semana durante los dos meses de } \\
\text { intervención }\end{array}$ \\
\hline 8 & $\begin{array}{l}\text { Modelo Demanda Control } \\
\text { de Karasek }\end{array}$ & Diseño de evaluación de un grupo con pretest y postest. \\
\hline
\end{tabular}

\section{En relación con los FRPSL que fueron intervenidos}

La Tabla 7 presenta los FRPSL Intralaboral intervenidos dentro de los estudios incluidos en la revisión. Es importante tener en cuenta que en los artículos en ocasiones se les dio un nombre diferente a los FRPSL intervenidos, en estos casos los investigadores realizaron una discusión sobre en cuál de las dimensiones establecidas por la Batería de Ministerio de la Protección Social se podía ubicar.

Se encontró solo una coincidencia en dos artículos puesto que se enfocan en generar oportunidades de desarrollo y uso de habilidades y destrezas, ofreciendo herramientas para que estas condiciones se cumplan en el trabajo. Estos dos artículos tenían en común que buscaban incrementar el empoderamiento y el conocimiento del puesto de trabajo y por lo tanto el dominio de la tarea, así se infirió que estaban enfocados a la misma dimensión del dominio control sobre el trabajo (Ministerio de la Protección Social., 2010).

Por otra parte, el tercer artículo buscó mejorar el control y la autonomía sobre el trabajo, interviniendo la toma de pausas, la decisión del orden en que se realiza el trabajo y la cantidad de trabajo que se realiza en la jornada laboral. Un cuarto artículo estuvo orientado a intervenir las relaciones sociales en el trabajo, generando un ambiente que permitiera la integración del grupo, confianza y colaboración entre los compañeros.

Tres artículos se enfocaron en condiciones que se ubican en el dominio demandas del trabajo, y dentro del mismo apuntaron a diferentes dimensiones; así uno se enfocó a las demandas ambientales y de esfuerzo físico, otro apuntó a intervenir las demandas cuantitativas de tiempo por ejemplo, donde el tiempo para la labor no alcanza o no hay tiempo para realizar las pausas y otro se refirió a las demandas emocionales, debido a que la población trabajadora debía enfrentarse a usuarios enojados, preocupados, tristes, enfermos, donde se podrían presentar agresiones de los usuarios o se debían mostrar sentimientos contrarios a los que se tenían.

El octavo artículo no evidenciaba claridad frente al factor de riesgo intervenido con el estudio. En los objetivos los autores planteaban que buscaban reducir los FRPSL que pueden llegar a causar el síndrome de burnout pero era ambiguo y no se concretaban claramente este aspecto. 
Tabla 7. FRPSL objeto de las intervenciones y tipos de intervenciones realizadas

\begin{tabular}{|c|c|c|c|}
\hline $\begin{array}{l}\text { Código } \\
\text { Artículo }\end{array}$ & Autores & $\begin{array}{c}\text { FRPS Intralaboral } \\
\text { objeto de intervención }\end{array}$ & Intervención \\
\hline 1 & $\begin{array}{l}\text { David M. DeJoy, Mark G. Wilson, } \\
\text { Robert J. Vandenberg, Allison L. } \\
\text { McGrath-Higgins and C. Shannon } \\
\text { Griffin-Blake }\end{array}$ & $\begin{array}{l}\text { Relaciones sociales en el } \\
\text { trabajo }\end{array}$ & $\begin{array}{l}\text { Creación de grupos participativos } \\
\text { paraformulación deintervenciones } \\
\text { específicas }\end{array}$ \\
\hline 2 & A. Häfner \& A. Stock & Demandas Cuantitativas & Entrenamiento/Capacitación \\
\hline 3 & $\begin{array}{l}\text { I.-L. Petterson, H. Donnersva, M. } \\
\text { Lagerstro, M. Toomingas }\end{array}$ & $\begin{array}{l}\text { Oportunidades de } \\
\text { Desarrollo y Uso de } \\
\text { Habilidades y destrezas }\end{array}$ & $\begin{array}{l}\text { Creación de grupos participativos } \\
\text { paraformulación deintervenciones } \\
\text { especificas }\end{array}$ \\
\hline 4 & $\begin{array}{l}\text { Afke J. M. B. Berkhout, Nicolle P.G. } \\
\text { Boumans, Frans J. N. Nijhuis, Gerard P. } \\
\text { J. Van Breukelen and Huda Huijer Abu- } \\
\text { Saad }\end{array}$ & $\begin{array}{l}\text { Control y Autonomía } \\
\text { sobre el trabajo }\end{array}$ & Reorganización del trabajo \\
\hline 8 & K. Calabro, T. A. Mackey, S.Williams & Demandas emocionales & $\begin{array}{l}\text { Diseño de instrumentos para } \\
\text { empoderar y mejorar la calidad del } \\
\text { trabajo }\end{array}$ \\
\hline 5 & J.E. Arnetza \& H. Hassona & $\begin{array}{l}\text { Oportunidades } \\
\text { Desarrollo y Uso de } \\
\text { Habilidades y destrezas }\end{array}$ & Entrenamiento/Capacitación \\
\hline 6 & $\begin{array}{l}\text { C. Cabrera, L.K. Ruiz, G.J. González, } \\
\text { M.G. Vega \& I. Valadez }\end{array}$ & $\begin{array}{lrr}\text { No fue posible } \\
\text { determinarlo }\end{array}$ & $\begin{array}{l}\text { Implementación de Pausas activas } \\
\text { en el lugar de trabajo }\end{array}$ \\
\hline 7 & $\begin{array}{l}\text { D.H de Castro, I.C. N. Sacco, L.E. } \\
\text { Rocha, C.A. de Bragança \& R.A. } \\
\text { Casarotto }\end{array}$ & $\begin{array}{l}\text { Demandas ambientales } \\
\text { del esfuerzo físico }\end{array}$ & \\
\hline
\end{tabular}

\section{Caracterización de los tipos de intervenciones realizadas}

En relación con los tipos de intervenciones utilizadas (Tabla 7) se encontró que la mayoría de ellas (cinco) fueron dirigidas hacia el individuo, es decir que buscaban las actividades se realizaban directamente con las personas. Entre estas actividades tres estudios se centraron en la capacitación o formación de las personas en aspectos relacionados con el trabajo, una en el desarrollo de instrumentos para empoderar y mejorar la calidad de vida de las personas en el trabajo y la última en la incorporación de pausas activas.

De los tres estudios restantes dos fueron realizados sobre el ambiente social del trabajo, creando grupos de participación para la formulación de acciones de mejora específicas de la sucursal o la institución en la que se encontraba cada equipo.

Un solo estudio se centró específicamente en el cambio de las condiciones del trabajo, planteando nuevas maneras de distribuir el trabajo, brindando mayor autonomía y enriqueciendo las tareas que se realizan en términos de variedad.

\section{Caracterización de los resultados obtenidos con las intervenciones realizadas}

La Tabla 8 especifica los aspectos sobre los que se midieron los resultados de las investigaciones y los efectos informados en las mismas. En relación los primeros (variables dependientes) se observó que todos los estudios no establecieron un solo efecto esperado, sino que evaluaron los cambios en un amplio número de condiciones de las personas y del trabajo. 
Siete de los estudios midieron los efectos de la intervención evaluando algunas de las dimensiones del dominio demandas del trabajo (demandas cuantitativas, demandas de carga mental, demandas emocionales, exigencias de la responsabilidad del cargo, demandas ambientales y de esfuerzo físico, demandas de la jornada de trabajo y consistencia de rol).

Siete de los estudios midieron los efectos considerando algunas de las dimensiones del dominio control sobre el trabajo (control y autonomía sobre el trabajo, oportunidades para el uso y desarrollo de habilidades y conocimientos, participación y manejo del cambio, claridad del rol y capacitación).

Tres buscaron medir los efectos sobre algunas de las dimensiones del dominio liderazgo y relaciones sociales en el trabajo (características del liderazgo, retroalimentación del desempeño, relaciones sociales en el trabajo).

Ninguno de los estudios incluidos en la investigación apuntó a FRPSL incluidos en el dominio recompensa (recompensas derivadas de la pertenencia a la empresa y reconocimiento y compensación).
Los efectos de las intervenciones se midieron en general, a través de la comparación de los resultados de las mediciones pre y post intervención, de los aspectos que se evaluaban en los cuestionarios aplicados. En cuanto a los efectos informados se evidencia en la mayoría de los estudios el mejoramiento de las percepciones sobre las dimensiones respecto a los dominios evaluados en los grupos intervenidos.

En dos de los estudios se reportó que no hubo mejoría en alguna de las características evaluadas. Tal es el caso de la disminución de oportunidades de aprendizaje en un estudio que evaluaba algunas dimensiones del dominio demandas del trabajo (Petterson et al., 2006).

Así mismo, en el estudio de Berkhout, Boumans, Nijhuis, Van Breukelen y Abu-Saad (2003) que evaluaba algunas dimensiones del dominio demandas del trabajo, control sobre el trabajo y liderazgo y relaciones sociales, se informaron inconsistencias en los resultados de los grupos intervenidos en cuanto el Apoyo Social y a la disminución del control percibido en el trabajo. 
Tabla 8 Efectos informados por las investigaciones encontradas

\begin{tabular}{|c|c|c|}
\hline Código & Aspectos en los que se miden los efectos & Efectos informados \\
\hline 1 & $\begin{array}{l}\text { Tres grupos de variables: } \\
\text { 1. Resultados proximales: diseño del } \\
\text { trabajo (carga de trabajo, control, } \\
\text { autonomía, claridad, condiciones del } \\
\text { ambiente, demandas físicas), clima } \\
\text { organizacional y trabajo futuro, resultados } \\
\text { del negocio } \\
\text { 2. Resultados intermedios: (ajuste } \\
\text { psicológico al trabajo) } \\
\text { 3. Resultados distales: Bienestar } \\
\text { (satisfacción, compromiso laboral, estrés, } \\
\text { autoeficacia para el trabajo e impacto del } \\
\text { trabajo) y salud percibida. }\end{array}$ & $\begin{array}{l}\text { 1. Resultados proximales: Mejorías en comunicación, participación } \\
\text { con otros, claridad de rol, contenido del trabajo, condiciones } \\
\text { ambientales, autonomía, programación del trabajo, oportunidades } \\
\text { de aprendizaje y justicia procedimental. En los grupos de control } \\
\text { las ventas descendieron y aumentaron las demandas ambientales. } \\
\text { 2. Resultados intermedios: teniendo en cuenta que Estados } \\
\text { Unidos al momento de la intervención se presentaba una crisis } \\
\text { económica disminuyó la satisfacción, compromiso y aumento } \\
\text { el estrés. No obstante, esta desmejora fue más significativa en el } \\
\text { grupo de control. } \\
\text { 3. Resultados distales: en las tiendas que se intervinieron mejoró la } \\
\text { salud percibida y la seguridad percibida en el trabajo. }\end{array}$ \\
\hline 2 & $\begin{array}{l}\text { - Manejo del tiempo } \\
\text { - Control percibido del tiempo } \\
\text { - Estrés percibido } \\
\text { - Auto organización } \\
\text { - Niveles de desempeño superior } \\
\text { - Tiempo gastado en una tarea importante } \\
\text { - Carga de trabajo percibida }\end{array}$ & $\begin{array}{l}\text { El grupo entrenado reportó mayores esfuerzos por mejorar } \\
\text { su gestión del tiempo y una mejora significativa en este } \\
\text { sentido; las estrategias de manejo del tiempo más usadas } \\
\text { fueron priorización y estructuración de la jornada de trabajo. } \\
\text { Los que recibieron el entrenamiento también hicieron mayor } \\
\text { seguimiento al nivel de logro de sus metas, percibieron más control } \\
\text { del tiempo y menor estrés después del entrenamiento. } \\
\text { Se encontró que a mayor control del tiempo disminuye la carga de } \\
\text { trabajo percibida. } \\
\text { No se encontraron diferencias significativas en auto organización } \\
\text { y tampoco en los niveles de desempeño superior. } \\
\text { El tiempo utilizado en una tarea importante aumentó en los dos } \\
\text { grupos después del pretest. }\end{array}$ \\
\hline 3 & $\begin{array}{l}\text { Carga de trabajo, carga física, recursos } \\
\text { del personal (para manejar los cambios, } \\
\text { apoyo de los directivos, apoyo de los } \\
\text { compañeros de trabajo, clientes y otros } \\
\text { relacionados, aprendizaje y desarrollo, } \\
\text { satisfacción laboral), salud y bienestar } \\
\text { (síntomas psicosomáticos, musculo } \\
\text { esqueléticos, síntomas de estrés, bienestar } \\
\text { y salud percibida), Recursos de salud } \\
\text { (afrontamientos, autoestima) calidad del } \\
\text { cuidado. }\end{array}$ & $\begin{array}{l}\text { En el grupo experimental: mejoría en la evaluación de la calidad } \\
\text { del cuidado, control sobre el trabajo y mejoramiento de la salud en } \\
\text { general, disminución en oportunidades de aprendizaje y tendencia } \\
\text { a incrementarse síntomas psicosomáticos y musculo esqueléticos. } \\
\text { Los que estaban en el hogar de los pacientes informaron un mayor } \\
\text { apoyo de compañeros que los que trabajaban en el hogar de } \\
\text { cuidados. } \\
\text { El grupo de los líderes innovadores percibió una mejora en la } \\
\text { calidad de su interacción con los familiares de los pacientes y } \\
\text { también en su autoestima. } \\
\text { Tendencia a cambiar de manera positiva, pero no significativas lo } \\
\text { cual atribuyen al tamaño pequeño del grupo. } \\
\text { No tuvieron compromiso de los grupos gerenciales. Por esta razón } \\
\text { se complicó medir los efectos en relación con este tema. }\end{array}$ \\
\hline
\end{tabular}




\section{Código Aspectos en los que se miden los efectos}

\section{Efectos informados}

Incremento en el número de pacientes asignados para darles un cuidado integral en los grupos experimentales después. Las salas de cuidado experimentales mostraron una mayor "desempeño orientado al paciente". En Autonomía laboral y responsabilidad no se reportó ningún efecto. En "Demandas del trabajo" se evidencio un incremento en los grupos experimentales y una disminución en una de las salas. Apoyo social: se encontraron resultados mixtos. En solo una de las salas experimentales se notó un aumento en el apoyo social.

Percepción de características de trabajo: Elprogramaplanteadoincluíaasignarlaresponsabilidaddecuidarpor Autonomía en el trabajo, demandas de completoa un númerodepacientes, estacondición no secumplió en trabajo, apoyo social.

varios de los grupos experimentales y cuando aumentaba la cantidad

4 Número de pacientes asignados, procesos de trabajo se volvía a trabajar igual que antes de la intervención. de enfermería por los que se responsabilizó Los planes de enfermería en los grupos experimentales estaban a la persona, asignación de tareas variadas y incompletos o no actualizados. La atención prevista no se comunicación.

correspondía plenamente con la realizada. Especialmente en momentos de alta presión de trabajo, se dio prioridad a la atención básica. Por otra parte, la delegación de las tareas de coordinación no había sido debidamente alcanzada. No se informaron los resultados esperados en relación con el aumento de la autonomía en el trabajo, disminución en las demandas del trabajo y mejoramiento del apoyo social. Los autores plantean que esto sucedió porque en las instituciones hubo un bajo compromiso de las directivas y, por lo tanto, no se realizaron los cambios establecidos en la manera de organizar el trabajo.

En el grupo intervenido: Después de la intervención se reportó una mejoría en la autoevaluación de conocimientos que los hacían más competentes en el trabajo.

Auto percepción de los conocimientos del El medio ambiente laboral, la participación, eficiencia, liderazgo, oficio; medio ambiente laboral (trabajo, el clima de trabajo, el rendimiento, la retroalimentación, las agotamiento y nivel de estrés) y utilización habilidades y la satisfacción en el trabajo, también mostraron de las herramientas de la caja. un mejoramiento significativo, aunque el nivel de agotamiento mostró un nivel de permanencia en la medición.

Se evidenció en el tiempo un aumento significativo de la utilización de instrumentos de la caja de herramientas.

El grupo uno (en el que se aplicó instrumento de medición de Burnout antes y después de desarrollar la intervención educativa) disminuyó en todos los indicadores: Disminución en el porcentaje de trabajadores con agotamiento emocional, despersonalización y falta de realización personal.

Los trabajadores que recibieron la intervención educativa mostraron más recursos y beneficios para evitar y/o controlar el desgaste laboral y la despersonalización al momento de analizar los indicadores del Síndrome de Burnout.

El programa psicoeducativo sensibilizó al personal para fomentar el desarrollo de estrategias para disminuir y controlar los estresores laborales que les afectan y que originan el Síndrome de Burnout. 


\begin{tabular}{|c|c|c|}
\hline Código & Aspectos en los que se miden los efectos & Efectos informados \\
\hline 7 & $\begin{array}{l}\text { Auto percepción de Malestar del Cuerpo } \\
\text { en tres áreas: S1 (cuello y hombros), S2 } \\
\text { (miembros superiores e inferiores) y S3 } \\
\text { (columna vertebral y glúteos). } \\
\text { Fatiga mental y física (cansancio percibido, } \\
\text { somnolencia, debilidad, dificultad de } \\
\text { concentración, memoria y dolor muscular). }\end{array}$ & $\begin{array}{l}\text { Grupo Experimental: tendencia hacia la disminución de la } \\
\text { frecuencia de las molestias en los segmentos del cuerpo S1 y S3 } \\
\text { y alivio de la fatiga, en comparación con el grupo control. El } \\
\text { segmento } S 2 \text { en el Grupo Control mostró también una tendencia } \\
\text { significativa a presentar mayores molestias. } \\
\text { El nivel de malestar, el número de segmentos del cuerpo con } \\
\text { molestias disminuyeron, y el porcentaje de segmentos dolorosos } \\
\text { se redujeron. }\end{array}$ \\
\hline 8 & $\begin{array}{l}\text {-Conocimiento (reconocimiento de } \\
\text { los niveles de crisis de los pacientes y las } \\
\text { respuestas adecuadas ante estos). } \\
\text {-Actitud (juicios subjetivos sobre el nivel } \\
\text { de acuerdo o desacuerdo para el uso de las } \\
\text { técnicas que le ensenan en el programa). } \\
\text {-Intención de conducta (probabilidad de } \\
\text { quela persona muestre los comportamientos } \\
\text { enseñados en el futuro). } \\
\text {-Autoeficacia (creencia que tiene la persona } \\
\text { en su habilidad para manejar las demandas } \\
\text { del trabajo). }\end{array}$ & $\begin{array}{l}\text { En el postestse encontraron mejorassignificativas en conocimiento, } \\
\text { la actitud, la auto-eficacia y la intención de conducta utilizar las } \\
\text { técnicas de entrenamiento. }\end{array}$ \\
\hline
\end{tabular}

\section{Discusión}

La pretensión inicial de esta investigación fue documentar los tipos de intervención primaria de los FRPSL y los efectos que esta evidencian para las organizaciones y el bienestar de los colaboradores. Este objetivo se cumplió de manera parcial dado que, teniendo en cuenta el amplio número de artículos revisados inicialmente y la escasa cantidad seleccionada finalmente, se puede afirmar son pocos los que reportan intervenciones y la gran mayoría de ellas están dirigidas a la prevención secundaria, o sea al tratamiento de los síntomas o de las alteraciones que se derivan de los FRPSL, especialmente a los síntomas de estrés, o también orientadas a la intervención de los factores de riesgo psicosocial individual. Esto podría indicar que aún existe poco interés por intervenir los FRPSL y que se ha buscado más intervenir las consecuencias de estos que los factores de riesgos psicosocial intralaboral. No obstante, también podría evidenciar que los encargados de la gestión de estos aspectos suelen encontrarse con limitaciones de tiempo o recursos en las organizaciones para implementación de acciones de mejora y que los efectos de estas pueden ser difíciles de documentar.

Tales hallazgos son coherentes con lo que se ha documentado ampliamente en la literatura acerca de la escasez de la intervención (Instituto Nacional de Seguridad e Higiene en el Trabajo, 2009), el enfoque reactivo más que preventivo (Instituto Sindical del Trabajo Ambiente y Salud, 2010) y la atención en el cambio de las condiciones disposicionales de los trabajadores o factores de riesgo psicosocial individual (Schnall, Dobson \& Rosskam, 2011;Lamontagne et al., 2010).

No obstante, también es importante tener en cuenta que el escaso número de reportes de intervención no necesariamente señala que al interior de las organizaciones no se realicen acciones para la reducción de los FRPSL, simplemente podría relacionarse con el hecho de que estas experiencias no se sistematizan y muchas veces no se llegan a conocer más allá de la organización en la que fueron hechas. 
Con el fin de indagar por este tipo de experiencias no publicadas, en España, el Instituto Nacional de Seguridad e Higiene en el trabajo realizó una selección de nueve experiencias exitosas de intervención (Pivaral et al., 2009) encontrándose fortalezas importantes en estos estudios. Seguramente si se realizaran esfuerzos similares sería posible identificar con mayor claridad hasta qué punto se están interviniendo los FRPS en las organizaciones.

El hecho de que la mayoría de estudios se hayan realizado en Europa puede estar relacionado con la existencia en estos países de una mayor normatividad, más antigua, y un mayor interés por la intervención y, especialmente, por la prevención en materia de FRPSL (Schnall, Dobson \& Rosskam, 2011), que en Latinoamérica. Tal es el caso de la directiva de la Unión Europea de 1989 relativa a la aplicación de medidas para promover la mejora de la seguridad y de la salud de los trabajadores que, establece además de que los empleadores deben evitar los riesgos y combatirlos en su origen y, de aliviar el trabajo monótono que se realiza a un ritmo predeterminado, para así reducir los efectos en la salud (Joyce, Pabayo, Critchley Julia, \& Bambra, 2010).

En los análisis por oficio y sector de la economía se encontró una clara tendencia a que la mayoría de las intervenciones se hayan realizado con personal asistencial en salud. Esto puede deberse a que, por las características propias de su trabajo, este personal generalmente está expuesto a altas demandas, especialmente de tipo emocional, y a extensas jornadas laborales, lo cual los hace una población muy vulnerable al estrés, al burnout y a enfermedades relacionadas con estos fenómenos (D’Amato \& Zijlstra, 2003). Otra explicación posible para este resultado puede ser que la mayoría de los investigadores interesados por este tema trabajan en el área de la salud y, por ende, pueden visualizar más fácilmente estos fenómenos en esta población.

También se encontró una intervención realizada con operadores de centros de llamadas los cuales se caracterizan por requerir posturas mantenidas, movimientos repetitivos, laborar en fines de semana o en horario nocturno y con escasa posibilidad de tomar pausas durante la jornada laboral. Sin embargo, no se encontraron estudios realizados en otros oficios identificados como de alto riesgo en el desarrollo de trastornos derivados del estrés como los maestros y fuerzas armadas (D’Amato \& Zijlstra, 2003).

El hallazgo de un estudio enfocado exclusivamente en población femenina puede deberse a que en el área de la salud, es común encontrar una predominancia de las mujeres en las profesiones relacionadas, tales como enfermería y trabajo social (Hofstede, 2004). No obstante, la mayoría de los estudios se realizaron con muestras mixtas, esto se puede relacionar con el hecho de que en la literatura no se han encontrado resultados concluyentes sobre la manera diferencial como los FRPSL afectan a los hombres y a las mujeres (D’Amato \& Zijlstra, 2003).

La totalidad de los estudios finalmente seleccionados fueron realizados con trabajadores urbanos, esto podría relacionarse con el hecho de que la mayoría de los estudios se hicieron en países industrializados, en los que, según la $\mathrm{FAO}$, menos del 5\% de la mano de obra se emplea en agricultura (FAO Food and Agriculture Organization of the United Nations, 2016). Esto no quiere decir que los trabajadores rurales carezcan de importancia en el estudio de los FRPSL, por el contrario, representan un grupo laboral muy importante por su tamaño, cerca del $50 \%$ de los trabajadores en el mundo, por las condiciones de riesgo a las que suelen enfrentarse y por la desprotección que a menudo tienen, por tratarse de trabajos comúnmente informales y temporales, en relación con seguridad social y seguridad laboral.

Siete de las intervenciones se basaron en la teoría de Demanda Control de Karasek, tal vez porque ha tenido una amplia comprobación científica de su capacidad para predecir el estrés y las enfermedades derivadas de este (OMS, 
2010). A pesar de que algunas no describían este enfoque de manera explícita, se concibieron de tal manera que buscaban principalmente aumentar las posibilidades de control que las personas podían ejercer, más que buscar reducir las demandas propias del cargo. Esto puede deberse a que generalmente en las organizaciones se "naturalizan" los FRPSL (Instituto Sindical del Trabajo Ambiente y Salud, 2010), es decir, se asume que son propios de ciertos cargos y no se hace nada por buscar reducir las demandas que le implican a quienes los desempeñan. No obstante, es importante tener en cuenta que las personas que diseñan los trabajos los conciben de esta manera lo cual hace posible repensar su configuración.

También este resultado podría asociarse con el hecho de que una proporción importante de los estudios fueron realizados con personal asistencial en salud, quienes realizan una labor en la que se puede aplicar una excepción a lo planteado en el párrafo anterior sobre rediseñar los cargos para reducir la demanda. Generalmente estas son profesiones con una alta demanda emocional que es inherente a su labor y, por ende, lo que se puede hacer es aumentar la autonomía de las personas en su cargo o brindar herramientas para que, al enfrentarse a estas situaciones, su salud y bienestar no se vean afectados (Sauter et al., 2001).

En lo relativo a la evaluación de la calidad de los artículos se utilizó un instrumento orientado a investigación cuantitativa en salud pública y generalmente las intervenciones sociales incluidas en este estudio no obedecen necesariamente a los criterios de esta rama. Lo anterior podría estar incidiendo en que muchos de ellos fueron calificados con una calidad baja. Sin embargo, luego de revisar los instrumentos disponibles para tal fin se consideró como la mejor opción ya que las demás se enfocaban más a la epidemiologia encontrándose menos coincidencia con las investigaciones objeto de este trabajo. También se encontró una proporción importante de estudios que fueron calificados con un débil control de las variables que puede causar distorsión en los resultados obtenidos. El aspecto identificado como fortaleza en la mayoría de ellos fue la calidad de los instrumentos utilizados para las mediciones, por el contrario las deficiencias más marcadas se observaron en la representatividad de las muestras, los diseños utilizados y el cegamiento en la asignación de los grupos experimentales y de control.

Tres de los artículos que cumplieron los criterios de inclusión no contaron con grupo de control. Según Hernández et al. (2010), esta clase de diseños son comúnmente criticados puesto que no se realiza un control de otras variables que pueden estar incidiendo en los cambios de la independiente.

Sin embargo, es importante destacar que, a pesar de las dificultades metodológicas encontradas en los artículos finalmente seleccionados, estos constituyen un esfuerzo de los investigadores que los realizaron por contribuir al conocimiento científico y proporcionan evidencia para quienes se encargan de diseñar las intervenciones en las empresas.

En relación con los FRPSL intervenidos, según la clasificación colombiana (Ministerio de la Protección Social, 2010), para el proceso de selección de los artículos fue clave que los investigadores tuvieran claridad sobre el tipo de factor intervenido y el tipo de intervención, pues aun cuando se observaron intervenciones en todo tipo de FRPSL, la mayoría de ellas que se referían al tema dirigían sus esfuerzos a trabajar los FRPSL desde el individuo, no enfocados a la prevención primaria como lo indicaba desde un inicio el estudio.

Es importante indicar, como se afirmaba al inicio de esta discusión, que de la reducida cantidad de artículos encontrados que buscaban intervenir de manera primaria FRPS Intralaboral, en tres dimensiones de los cuatro dominios propuestos en la Batería para la Medición e Intervención de 
Riesgo Psicosocial Colombiana (Ministerio de la Protección Social, 2010). El hecho de que solo uno de los estudios esté orientado al dominio liderazgo y relaciones sociales en el trabajo señala que, a pesar de ser un factor de riesgo importante, generador de consecuencias (Sauter et al., 2001) como el estrés o conductas de acoso laboral, al parecer no se han generado las estrategias necesarias para hablar de intervenciones sobre este tipo de factores, en parte debido a la investigación escasa y a las limitaciones de tipo metodológico para su intervención (Caicoya, 2004).

$\mathrm{Si}$ el principal enfoque de intervención primaria sobre este tipo de factores de riesgo se enmarca desde los dominios de demandas del trabajo (Artículos con código 2, 7 y 8) y control sobre el trabajo (artículos con códigos 3, 4 y 5) es debido a que cada vez es más demostrable que las altas demandas del trabajo y el poco control sobre el mismo generan consecuencias graves como el estrés (Sauter et al., 2001) y otros efectos como desbalance trabajo-familia (OMS, 2010). Lo que demuestra que hay más esfuerzos por generar ese equilibrio desde las intervenciones de tipo primario dirigidas a intervenir la fuente generadora.

En lo referido a los tipos de intervenciones realizadas se halló que en la literatura, cuando se habla de las condiciones necesarias para que las intervenciones de los FRPSL sean exitosas, se contempla que es necesario dar participación a las personas en este proceso ya que son quienes directamente son afectadas por ellos (Pivaral et al., 2009). Esta condición se cumple en la mayoría de los estudios realizados, teniendo en cuenta que en cinco de ellos las actividades se realizan directamente con los individuos y otras dos crearon grupos de participación de las acciones de mejora lo cual también requiere que las personas participen de manera activa.

Las actividades de formación y capacitación han demostrado tener una eficacia moderada en el control de los FRPSL (Moncada i Lluís et al., 2005) y en la creación de entornos de trabajo saludables (Leka et al., 2004). No obstante, estas intervenciones deberían estar acompañadas de otro tipo de estrategias que busquen controlar las condiciones del trabajo que muchas veces constituyen por sí mismas la fuente de los FRPSL, pues a pesar de que las personas cuenten con los conocimientos necesarios para el manejo de las situaciones que se presenten en su trabajo, cuando están expuestas a condiciones desfavorables, terminaran por sufrir efectos negativos en su salud y bienestar (Lamontagne et al., 2010; Moncada i Lluís et al., 2005).

Además, en las situaciones en las que los trabajadores observan que los empleadores no están dispuestos a realizar cambios a nivel de la estructuración del trabajo, su disposición a la participación en las actividades y a implementar cambios en su comportamiento disminuyen (Instituto Sindical del Trabajo Ambiente y Salud, 2010), lo cual limita también el impacto de las intervenciones.

En relación con los resultados reportados por las investigaciones, es importante anotar que en cuanto al enfoque preventivo de los estudios desde los diseños de las investigaciones fue diverso en las intervenciones, aproximándose a lo que recomienda Kristensen (Caicoya, 2004), actuando a diferentes niveles entre los estudios: sobre los individuos, la interfaz del individuo organización y sobre la organización. Puesto que al observar donde se medirían los efectos la mayoría de los estudios (7 artículos) los querían encontrar tanto en las demandas del trabajo como en el control sobre el trabajo, indagando sobre las percepciones de las personas utilizando cuestionarios pretest y postest, lo que mostró voluntad por parte de las organizaciones a prevenir directamente la exposición a los factores de riesgo mencionados (Caicoya, 2004).

Si solo tres de los artículos trataron de medir los efectos de los resultados sobre el liderazgo y las relaciones sociales se puede inferir que, desde la planeación no se propuso medir ese tipo de 
factores debido a que las intervenciones sobre tales asuntos desde la prevención requieren medidas administrativas que implican esfuerzos de las áreas estratégicas de la empresa. Es evidente que las organizaciones pueden tener eventualmente más recursos para invertir en el personal, sin embargo al momento de la intervención por medio de actividades de formación y capacitación a veces no se establecen principios respecto a este tipo de factores de riesgo ni directrices claras de formación para los jefes y colaboradores con el fin de que tengan las habilidades necesarias para la convivencia en las relaciones sociales (OMS, 2010).

El hecho de que todas las intervenciones hayan demostrado efectos positivos sobre algunos de los factores intervenidos puede indicar el éxito de las mismas; sin embargo, esta investigación no buscaba comprobar su efectividad, sino la evidencia de esas intervenciones primarias sobre FRPSL. También demuestra, como lo sostenía Karasek que si se incrementa la armonía entre las demandas del trabajo y el control sobre el trabajo, las reacciones sobre la salud, el bienestar y la satisfacción de las personas pueden verse favorecidas significativamente (Toro et al., 2010).

Se encontraron dos intervenciones en las que no se lograron los resultados esperados (códigos de artículos 3 y 4). Revisando los artículos se pudo haber dificultado la obtención de los resultados porque se requería un alto compromiso de la dirección en relación con cambiar la manera como se distribuían las tareas y la autonomía que los líderes daban a las personas. Sin embargo hubo escasa voluntad en este sentido y, por lo tanto, no se cumplía con los requerimientos de la intervención, por ejemplo, en momentos de alta demanda del trabajo se recurría a la manera anterior de distribuir las tareas. Desde la teoría, este tipo de intervenciones que buscan cambiar los aspectos del trabajo que representan un FRPSL son los que mayor efectividad reportan (Instituto Sindical del Trabajo Ambiente y Salud, 2010), pero se confirma la resistencia de las organizaciones a cambiar las condiciones del trabajo y esperar que sean los trabajadores quienes cambien para afrontar de manera más efectiva las situaciones (Instituto Nacional de Seguridad e Higiene en el Trabajo, 2009).

\section{Limitaciones}

Cada base de datos tenía una manera diferente de realizar el rastreo, y al no ser posible guardar los avances en las búsquedas realizadas, se debían ingresar de nuevo los datos con todos los controles y eso requirió buena parte de tiempo de la investigación.

Se encontró además, dificultad para acceder a algunos artículos debido a que no se encontraban disponibles para los usuarios de la Universidad de Antioquia y su costo era elevado.

\section{Recomendaciones}

1. Realizar una revisión sistemática a nivel nacional o latinoamericano que incluya otras fuentes de información tales como literatura gris, experiencias de empresas, entre otras, de tal manera que se pueda llegar a conocer con mayor certeza el nivel de desarrollo que la prevención de los FRPSL ha tenido en nuestro medio.

2. En las intervenciones de los FRPSL que se realicen en adelante buscar que tengan un enfoque más proactivo que reactivo y adoptar el enfoque de control de los riesgos que se maneja en otras áreas de la salud ocupacional, en la medida de los posible, en la fuente más que en el medio o las personas.

3. Como componente esencial en las intervenciones que se vayan a realizar siempre se debe contar con el compromiso de la dirección de las organizaciones. De lo contrario se realizarán actividades con una capacidad muy limitada para producir cambios favorables para la salud y el bienestar de los trabajadores. 
4. En investigaciones posteriores es importante que se busque intervenir o identificar experiencias de intervención exitosas en trabajadores de otros oficios que, en la literatura científica, han sido identificados como de alto riesgo psicosociales, tales como las fuerzas militares y los docentes.

5. También sería conveniente profundizar en el tema del dominio de las recompensas en el trabajo, dado que en la literatura ampliamente se han identificado los efectos de este tipo de FRPSL y no se identificaron intervenciones de prevención primaria a este nivel.

\section{Referencias}

Afke J. M. B. Berkhout, Nicolle P. G. Boumans, Frans J. N. Nijhuis, Gerard P. J. Van Breukelen, \& Huda Huijer Abu-saad. (2003). Effects of resident-oriented care on job characteristics of nursing caregivers. Work \& Stress, 17(4), 337-353. http://doi. org/10.1080/02678370 310001647645

Aguilar, M., \& Rentería, E. (2009). Psicología del trabajo y las organizaciones. Bogotá: Universidad Santo Tomás.

Arnetz, J. E., \& Hasson, H. (2007). Evaluation of an educational "toolbox" for improving nursing staff competence and psychosocial work environment in elderly care: Results of a prospective, nonrandomized controlled intervention. International Journal of Nursing Studies, 44(5), 723-735. http:// doi.org/10.1016/j.jinurstu.2006.01.012

Bambra, C. (2009). Real world reviews: A beginner's guide to undertaking systematic reviews of public health policy interventions. Journal of Epidemiology and Community Health, 1-15. http://doi.org/10.1136/ jech.2009.088740

Beltrán, O. A. (2005). Revisiones sistemáticas de la literatura. Rev. Colombiana de Gastroenterología, 20(1), 6069. Retrieved from www.gastrocol.com/file/Revista/ v20n1a09.pdf

Caicoya, M. (2004). Dilemas en la evaluación de riesgos psicosociales. Arch Prev Riesgos Labor, 7(3), 109118.

Calabro, K., Mackey, T. A., \& Williams, S. (2002)., 3-15. To Prevent And Manage Patient Violence

Carver, C. S., Scheier, M. F., \& Weintraub, J. K. (1989). Assessing coping strategies: A theoretically based approach. Journal of Personality and Social Psychology, 56(2), 267-283. http://doi. org/10.1037/0022-3514.56.2.267

Cochrane, C. (2016). Cochrane Iberoamérica. Retrieved from http://es.cochrane.org/es

Cuadra-Peralta, A.; Veloso-Besio, C.; Puddu-Gallardo, G.; Salgado-García, P. \& Peralta-Montecinos, J. (2012). Impacto de un Programa de Psicología Positiva en Sintomatologia Depresiva y Satisfaccion Vital en Adultos Mayores . Psicologia: Reflexão E Crítica, 25(4), 644-652. http://doi.org/10.1590/S010279722012000400003

D’Amato, A., \& Zijlstra, F. R. (2003). Occupational stress: A review of the literature relating to mental health. Stress Impact. Surrey: University of Surrey.

DeJoy, D. M., Wilson, M. G., Vandenberg, R. J., McGrathHiggins, A. L., \& Griffin-Blake, C. S. (2010). Assessing the impact of healthy work organization intervention. Journal of Occupational and Organizational Psychology, 83(1), 139-165. http:// doi.org/10.1348/096317908X398773

Dela Coleta, J.A. Atribução de causalidade: Teoría, pesquisa e aplicaçães. 2a. Ed. Sao Paulo: Cabral Editora; 2006.

Departamento Administrativo Nacional de Estadística (DANE). (2016). Retrieved from http://www.dane. gov.co/\#twoj_fragment 1-4.

FAO Food and Agriculture Organization of the United Nations. (2016). Retrieved from http://www.fao.org/ americas $/$ noticias/en $/$ ?page $=62 \& i p p=20 \&$ tx_dynalist_pi1\%5Bpar\%5D=YToxOntzOjE6IkwiO3M6MToiMiI7fQ\%3D\%3D

Häfner, A., \& Stock, A. (2010). Time Management Training and Perceived Control of Time at Work. Journal of Psychology, 144(5), 429-447. Retrieved from http://search.ebscohost.com/login.aspx?dir ect $=$ true $\& \mathrm{db}=$ aph $\& A N=52341878 \&$ site $=$ ehost live\&scope $=$ site

Hernández, R., Fernández, C., \& Baptista, P. (2010). Metodología de la investigación. México: McGrawHill.

Herruzo Cabrera, J., \& Moriana Elvira, J. A. (2004). Estrés y burnout en profesores. International Journal of Clinical and Health Psychology, 4(3), 597-621. Retrieved from http://dialnet.unirioja.es/servlet/articulo $?$ codigo $=990187 \&$ info $=$ resumen $\&$ idioma $=$ ENG

Hofstede, G. (2004). Cultures and organizations software of the mind. Mc Graw Hill (Vol. 23). http://doi.org/10.1177/017084068400500423 $10.1177 / 017084069401500308$ 
Instituto Nacional de Seguridad e Higiene en el Trabajo. (2009). Experiencias en intervención psicosocial. Más allá de la evaluación del riesgo.

Instituto Sindical del Trabajo Ambiente y Salud. (2010). Manual del método para la evaluación y prevención de los riesgos psicosociales para empresas con 25 o más trabajadores y trabajadoras (Vol. 21).

Jané-llopis, E. (2004). La eficacia de la Promoción de la salud mental y la Prevención de los trastornos mentales. Revista de La Asociación Española de Neuropsiquiatría, 89(67), 67-77. Retrieved from http://scielo.isciii.es/scielo.php?pid=S021157352004000100005\&script=sci_arttext

Joyce, K., Pabayo, R., Critchley Julia, A., \& Bambra, C. (2010). Flexible working conditions and their effects on employee health and wellbeing. Cochrane Database of Systematic Reviews, (2). http://doi. org/10.1002/14651858.CD008009.pub2

Lacaze, D. H. D. C., Sacco, I. D. C. N., Rocha, L. E., Pereira, C. A. D. B., \& Casarotto, R. A. (2010). Stretching and joint mobilization exercises reduce call-center operators' musculoskeletal discomfort and fatigue. Clinics (Sao Paulo, Brazil), 65(7), 657-662. http:// doi.org/10.1590/S1807-59322010000700003

Lamontagne, A. D., Keegel, T. G., \& Centre, M. (2010). What organisational / employer level interventions are effective for preventing and treating occupational stress ? Health (San Francisco), (December), 1-19.

Lamontagne, A. D., Keegel, T., Louie, A. M., Ostry, A., \& Landsbergis, P. A. (2007). A systematic review of the job-stress intervention evaluation literature, 19902005. International Journal of Occupational and Environmental Health, 13(3), 268-280. http://doi. org/10.1179/oeh.2007.13.3.268

Lazcano-Ponce, E., Salazar-Martínez, E., Gutiérrez-Castrell, P., Angeles-Llerenas, A., Hernández-Garduo, A., \& Viramontes, L. (2004). Ensayos clínicos aleatorizados: Variantes, métodos de aleatorización, análisis, consideraciones políticas y regulación. Salud Publica de Mexico, 46(6), 559-584. http://doi. org/10.1590/S0036-36342004000600012

Leka, S., Griffiths, A., \& Cox, T. (2004). La organización del trabajo y el estrés. Serie Protección de La Salud de Los Trabajadores de Los Trabajadores Trabajadores No 3, 1 - 37. Retrieved from http://www.who.int/ occupational_health/publications/pwh3sp.pdf

Llorens, S., Bakker, A. B., Schaufeli, W. B., \& Salanova, M. (2007). "Testing the robustness of the job demandsresources model”: Erratum. International Journal of
Stress Management, 14(2), 224-225. http://doi. org/10.1037/1072-5245.14.2.224

Martínez, J. C. (2008). Clima Organizacional: Una Opción Empresarial O Una Obligación Legal. Revista Interamericana de Psicología Ocupacional, 27(1), $58-60$.

Martínez-Losa, J. \& Bestratén, M. (2010). Desarrollo de competencias y riesgos psicosociales ( I ). Notas Técnicas de Prevención.

Ministerio de la Protección Social. (2008). Resolución 2646. 2008. Retrieved from http://copaso.upbbga.edu. co/legislacion/resolucion_2646_2008_Factores de Riesgo Sicosocial.pdf

Ministerio de la Protección Social. (2010). Batería de instrumentos para la evaluación de factores de riesgo psicosocial.

Moncada i Lluís, S., Llorens Serrano, C., S Kristensen, T., \& Vega Martínez, S. (2005). Nota Técnica de Prevención (703). El Método COPSOQ (ISTAS21, PSQCAT21) de Evaluación de Riesgos Psicosociales.

OMS. (2010). Entornos Laborales Saludables : Fundamentos y Modelo de la OMS. Contextualización, prácticas y literatura de apoyo., 144.

Petterson, I. L., Donnersväärd, H. A. A., Lagerströöm, M., \& Toomingas, A. (2006). Evaluation of an intervention programme based on empowerment for eldercare nursing staff. Work and Stress, 20(4), 353-369. http://doi.org/10.1080/02678370601070489

Pivaral, C.E. C., Cruz, L. K. R., Pérez, G.J. G., López, M. G. V., \& Figueroa, I. V. (2009). Efecto de una intervención psicoeducativa para disminuir el síndrome Burnout en personal de confianza de la comisión federal de electricidad. Salud Mental, 32(3), 215-221.

Salanova, M. (2009). Psicología de la salud ocupacional. Madrid: Síntesis,.

Sauter, S. L., Murphy, L. R., Hurrell, J. J., \& Levi, L. (2001). Factores psicosociales y de organización. Enciclopedia de Salud Y Seguridad En El Trabajo, 73.

Schnall, P. L., Dobson, M., \& Rosskam, E. (2011).

Trabajo no saludable: Causas, consecuencias, curas. Ediciones Uniandes.

Thomas, H. (2003). Quality assessment tool for quantitative studies. Effective Public Health Practice Project. McMaster ..., 2-5. Retrieved from http://scholar. google.com/scholar?hl=en\&btnG=Search\&q=intitle:QUALITY+ASSESSMENT+TOOL + FOR+QUANTITATIVE+STUDIES\# 1 
Toro, F., Londoño, M.E., Sanin, A., \& Valencia, M. (2010). Modelo analítico de factores psicosociales en contextos laborales. Revista Interamericana de Psicología Ocupacional, 29(2), 95-137.
Villalobos, G. H. (2007). Diseño de un Sistema de Vigilancia Epidemiológica de Factores de Riesgo Psicosocial en el Trabajo. 